\title{
New Aspects of Bloch Model Associated with Fractal Fractional Derivatives
}

https://doi.org/10.1515/nleng-2021-0026

Received Mar 14, 2021; accepted Sep 20, 2021.

\begin{abstract}
To model complex real world problems, the novel concept of non-local fractal-fractional differential and integral operators with two orders (fractional order and fractal dimension) have been used as mathematical tools in contrast to classical derivatives and integrals. In this paper, we consider Bloch equations with fractalfractional derivatives. We find the general solutions for components of magnetization $\mathcal{M}=\left(M_{u}, M_{v}, M_{w}\right)$ by using descritization and Lagrange's two step polynomial interpolation. We analyze the model with three different kernels namely power function, exponential decay function and Mittag-Leffler type function. We provide graphical behaviour of magnetization components $\mathcal{M}=\left(M_{u}, M_{v}, M_{w}\right)$ on different orders. The examination of Bloch equations with fractal-fractional derivatives show new aspects of Bloch equations.
\end{abstract}

\section{Introduction}

We consider the Bloch equations as [1]:

$$
\begin{aligned}
& \frac{d M_{u}(t)}{d t}=\vartheta_{0} M_{v}(t)-\frac{M_{u}(t)}{\lambda_{2}} . \\
& \frac{d M_{v}(t)}{d t}=-\vartheta_{0} M_{u}(t)-\frac{M_{v}(t)}{\lambda_{2}} . \\
& \frac{d M_{w}(t)}{d t}=\frac{M_{0}-M_{w}(t)}{\lambda_{1}} .
\end{aligned}
$$

The above equations have been given by Flex Bloch (1946), are a group of microscopic equations applied in several branches of science. These equations are mainly used in

\footnotetext{
*Corresponding Author: Ali Akgül, Department of Mathematics, Art and Science Faculty, Siirt University, TR-56100 Siirt, Turkey, E-mail: aliakgul@siirt.edu.tr

Ishfaq Ahmad Mallah, Department of Mathematics, Maulana Azad National Urdu university, Hyderabad-500032, India,

E-mail: ishfaqmalla@gmail.com

Subhash Alha, Department of Mathematics, Maulana Azad National Urdu university, Hyderabad-500032, India,

E-mail: subhashalha@manuu.edu.in
}

nuclear magnetic resonance (NMR), to quantify the nuclear magnetization $\mathcal{M}=\left(M_{u}, M_{v}, M_{w}\right)$, where $M_{u}(t)$, $M_{v}(t)$ and $M_{w}(t)$ denote the system magnetization in $u$, $v$ and $w$ component respectively. Also $M_{0}$ represents the equilibrium magnetisation, $\vartheta_{0}$ given by $\vartheta_{0}=y N_{0}$ is resonant frequency. Here, $N_{0}$ is the static magnetic field in zcomponent. Furthermore, $\lambda_{1}$ and $\lambda_{2}$ respectively represent spin-lattice and spin-spin relaxation time.

Nowadays, in the area of fractional calculus, various types of differential operators are being used. Initially, Chen-Wen [2] proposed the differential operator known as fractal derivative. It was revealed in various papers [310] that the fractal-differential operator studies the natural phenomena more correctly than the classical derivative due to the presence of fractal dimension parameter. More recently, Caputo and Fabrizio have introduced the fractional derivative with non-sigular kernel [11]. In the same context, new differential operators called fractalfractional derivatives with non-local and non-singular kernels involving two orders, the fractional order and the fractal dimension, were introduced by Atangana [12]. They are convolution of a given function and the fractional kernels having the form as power law, exponential decay law and the Mittag-Leffler function. The Reimann-Liouville and Caputo correspond to power law, Caputo-Fabrizio represents the exponential decay law and the Atangana-Baleanu corresponds to Mittag-Leffler function [11, 13, 14]. These operators show remarkable progress in modeling due to their higher accuracy and strong reliability $[15,16]$.

In literature, there exits various approaches to derive approximate solution of Bloch equations [17-19]. Recently, a mathematical model on Bloch equations with composite fractional derivative was solved by some authors [1].

In the present paper, we apply the concept of fractional fractal derivative of orders $(\alpha, \tau)$ to study new aspects of fractional Bloch equations. For that, we replace the classical derivatives in Bloch equations by the derivatives having kernels as power law, exponential decay law and the Mittag-Leffler law.

The work in this paper is organized as follows: In Section 2, some useful definitions related to fractal-fractional derivatives are given. A numerical scheme has been presented for non-linear fractional differential equations having non-local and non-singular kernel in Section 3. Anal- 
ysis of the Bloch equations related to fractal-fractional derivatives using the exponential decay law is given in Section 4. Moreover, Section 5 provides figures to show numerical simulation and comparison of results for the solution of fractional Bloch equations. In last section, the conclusion is presented.

\section{Some definitions and preliminaries for fractal fractional derivatives}

Definition 2.1. Let the function $\phi(t)$ be continuous and fractal differentiable on some open interval $(c, d)$ with order $\tau$. Then, the fractal-fractional derivative of $\phi$ in Riemann-Liouville sense with order $\alpha$ having the power law type kernel is defined as [20]:

${ }_{c}^{F F P} D_{t}^{\alpha, \tau} \phi(t)=\frac{1}{\Gamma(1-\alpha)} \frac{d}{d t^{\tau}} \int_{c}^{t}(t-s)^{-\alpha} \phi(s) d s, 0<\alpha, \tau \leq 1$,

where,

$$
\frac{d \phi(s)}{d s^{\tau}}=\lim _{t \rightarrow s} \frac{\phi(t)-\phi(s)}{t^{\tau}-s^{\tau}}
$$

Definition 2.2. Let the function $\phi(t)$ be continuous and fractal differentiable on some open interval $(c, d)$ with order $\tau$. Then, the fractal-fractional derivative of $\phi$ in Riemann-Liouville sense with order $\alpha$ having the exponential decay type kernel is defined as [20]:

$$
\begin{aligned}
& { }_{c}^{F F E} D_{t}^{\alpha, \tau} \phi(t)=\frac{M(\alpha)}{1-\alpha} \frac{d}{d t^{\tau}} \int_{c}^{t} \exp \left(\frac{-\alpha}{1-\alpha}(t-s)\right) \phi(s) d s, \\
& 0<\alpha, \tau \leq 1 .
\end{aligned}
$$

Definition 2.3. Let the function $\phi(t)$ be continuous and fractal differentiable on some open interval $(c, d)$ with order $\tau$. Then, the fractal-fractional derivative of $\phi$ in Riemann-Liouville sense with order $\alpha$ having the generalized Mittag-Leffler type kernel is defined as [20]:

$$
\begin{aligned}
& { }_{c}^{F F M} D_{t}^{\alpha, \tau} \phi(t)=\frac{A B(\alpha)}{1-\alpha} \frac{d}{d t^{\tau}} \int_{c}^{t} E_{\alpha}\left(\frac{-\alpha}{1-\alpha}(t-s)^{\alpha}\right) \phi(s) d s \\
& 0<\alpha, \tau \leq 1
\end{aligned}
$$

where, $A B(\alpha)=1-\alpha+\frac{\alpha}{\Gamma(\alpha)}$.

Definition 2.4. The fractal-fractional integral of order $\alpha$ of a function $\phi(t)$, continuous in some open interval $(c, d)$ corresponding to the fractal fractional derivative having power law type kernel is given as [20]:

$$
{ }_{0}^{F F P} I_{t}^{\alpha, \tau} \phi(t)=\frac{\tau}{\Gamma(\alpha)} \int_{0}^{t}(t-s)^{\alpha-1} s^{\tau-1} \phi(s) d s
$$

Definition 2.5. The fractal-fractional integral of order $\alpha$ of a function $\phi(t)$, continuous in some open interval $(c, d)$ corresponding to the fractal fractional derivative having exponential decay type kernel is given as[20]:

$$
{ }_{0}^{F F E} I_{t}^{\alpha, \tau} \phi(t)=\frac{\alpha \tau}{M(\alpha)} \int_{0}^{t} s^{\tau-1} \phi(s) d s+\frac{\tau(1-\alpha) t^{\tau-1}}{M(\alpha)} \phi(t) .
$$

Definition 2.6. The fractal-fractional integral of order $\alpha$ of a function $\phi(t)$, continuous in some open interval $(c, d)$ corresponding to the fractal fractional derivative having Mittag-Leffler type kernel is given as [20]:

$$
\begin{aligned}
& { }_{0}^{F F M} I_{t}^{\alpha, \tau} \phi(t)= \\
& \frac{\alpha \tau}{A B(\alpha)} \int_{0}^{t}(t-s)^{\tau-1} s^{\alpha-1} \phi(s) d s+\frac{\tau(1-\alpha) t^{\tau-1}}{A B(\alpha)} \phi(t) .
\end{aligned}
$$

\section{Numerical Scheme}

In this section, we write a numerical scheme newly constructed by Atangana and Toufik [21] for non-linear fractional differential equations having non-local and nonsingular kernel. Let us consider the following fractional differential equation as:

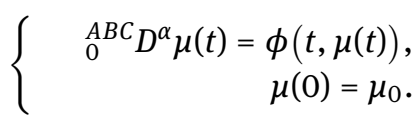

By applying the concept of fundamental theorem of fractional calculus, (3.1) gives

$$
\begin{aligned}
& \mu(t)-\mu(0)=\frac{(1-\alpha)}{A B C(\alpha)} \phi(t, \mu(t)) \\
& +\frac{\alpha}{\Gamma(\alpha) \times A B C(\alpha)} \int_{0}^{t} \phi(s, \mu(s))(t-s)^{\alpha-1} d s .
\end{aligned}
$$

Hence at $t=t_{n+1}, n=0,1,2, \ldots$, the above equation can be redefined as 


$$
\begin{aligned}
\mu\left(t_{n+1}\right)-\mu(0) & =\frac{(1-\alpha)}{A B C(\alpha)} \phi\left(t_{n}, \mu\left(t_{n}\right)\right)+\frac{\alpha}{\Gamma(\alpha) \times A B C(\alpha)} \int_{0}^{t_{n+1}} \phi(s, \mu(s))\left(t_{n+1}-s\right)^{\alpha-1} d s \\
& =\frac{(1-\alpha)}{A B C(\alpha)} \phi\left(t_{n}, \mu\left(t_{n}\right)\right)+\frac{\alpha}{\Gamma(\alpha) \times A B C(\alpha)} \sum_{j=0}^{n} \int_{t_{j}}^{t_{j+1}} \phi(s, \mu(s))\left(t_{n+1}-s\right)^{\alpha-1} d s .
\end{aligned}
$$

The function $\phi(s, \mu(s))$ with in the interval $\left[t_{j}, t_{j+1}\right]$ can be approximated by the Lagrange's two-step polynomial interpolation as:

$$
\begin{aligned}
P_{j}(s) & =\frac{s-t_{j-1}}{t_{j}-t_{j-1}} \phi\left(t_{j}, \mu\left(t_{j}\right)\right)-\frac{s-t_{j}}{t_{j}-t_{j-1}} \phi\left(t_{j-1}, \mu\left(t_{j-1}\right)\right) \\
& =\frac{\phi\left(t_{j}, \mu\left(t_{j}\right)\right)}{h}\left(s-t_{j-1}\right)-\frac{\phi\left(t_{j-1}, \mu\left(t_{j-1}\right)\right)}{h}\left(s-t_{j}\right) \simeq \frac{\phi\left(t_{j}, \mu_{j}\right)}{h}\left(s-t_{j-1}\right)-\frac{\phi\left(t_{j-1}, \mu_{j-1}\right)}{h}\left(s-t_{j}\right) .
\end{aligned}
$$

Using the above approximation in (3.3), we have:

$$
\begin{aligned}
\mu_{n+1} & =\mu_{0}+\frac{(1-\alpha)}{A B C(\alpha)} \phi\left(t_{n}, \mu\left(t_{n}\right)\right) \\
& +\frac{\alpha}{\Gamma(\eta) \times A B C(\alpha)} \sum_{j=0}^{n}\left(\frac{\phi\left(t_{j}, \mu_{j}\right)}{\alpha} \int_{t_{j}}^{t_{j+1}}\left(s-t_{j-1}\right)\left(t_{n+1}-s\right)^{\alpha-1} d s-\frac{\phi\left(t_{j-1}, \mu_{j-1}\right)}{\alpha} \int_{t_{j}}^{t_{j+1}}\left(s-t_{j}\right)\left(t_{n+1}-s\right)^{\alpha-1} d s\right) .
\end{aligned}
$$

To be simple:

$$
A_{\alpha, j, 1}=\int_{t_{j}}^{t_{j+1}}\left(s-t_{j-1}\right)\left(t_{n+1}-s\right)^{\alpha-1} d s
$$

and also:

$$
\begin{aligned}
& A_{\alpha, j, 2}=\int_{t_{j}}^{t_{j+1}}\left(s-t_{j}\right)\left(t_{n+1}-s\right)^{\alpha-1} d s . \\
& A_{\alpha, j, 1}=h^{\alpha+1} \frac{(n+1-j)^{\alpha}(n-j+2+\alpha)-(n-j)^{\alpha}(n-j+2+2 \alpha)}{\alpha(\alpha+1)} . \\
& A_{\alpha, j, 2}=h^{\alpha+1} \frac{(n+1-j)^{\alpha+1}-(n-j)^{\alpha}(n-j+1+\alpha)}{\alpha(\alpha+1)} .
\end{aligned}
$$

Now Integrating (3.6), (3.7), and then put them in (3.5) we get:

$$
\begin{aligned}
\mu_{n+1}=\mu_{0} & +\frac{(1-\alpha)}{A B C(\alpha)} \phi\left(t_{n}, \mu\left(t_{n}\right)\right)+\frac{\alpha}{A B C(\alpha)} \sum_{j=0}^{n}\left(\frac{h^{\alpha} \phi\left(t_{j}, \mu_{j}\right)}{\Gamma(\alpha+2)}\left((n+1-j)^{\alpha}(n-j+2+\alpha)-(n-j)^{\alpha}(n-j+2+2 \alpha)\right)\right. \\
& \left.-\frac{h^{\alpha} \phi\left(t_{j-1}, \mu_{i-1}\right)}{\Gamma(\alpha+2)}\left((n+1-j)^{\alpha+1}-(n-j)^{\alpha}(n-j+1+\alpha)\right)\right) .
\end{aligned}
$$

\section{Analysis with the Exponential-Decay Kernel}

We consider the standard Bloch equations as [1]:

$$
\begin{aligned}
& \frac{d M_{u}(t)}{d t}=\vartheta_{0} M_{v}(t)-\frac{M_{u}(t)}{\lambda_{2}} . \\
& \frac{d M_{v}(t)}{d t}=-\vartheta_{0} M_{u}(t)-\frac{M_{v}(t)}{\lambda_{2}} . \\
& \frac{d M_{w}(t)}{d t}=\frac{M_{0}-M_{w}(t)}{\lambda_{1}} .
\end{aligned}
$$


We replace the classical derivatives with the fractal-fractional derivatives and obtain:

$$
\begin{aligned}
& { }_{c}^{F F E} D_{t}^{\alpha, \tau} M_{u}(t)=\vartheta_{0} M_{v}(t)-\frac{M_{u}(t)}{\lambda_{2}} . \\
& { }_{c}^{F F E} D_{t}^{\alpha, \tau} M_{v}(t)=-\vartheta_{0} M_{u}(t)-\frac{M_{v}(t)}{\lambda_{2}} . \\
& { }_{c}^{F F E} D_{t}^{\alpha, \tau} M_{w}(t)=\frac{M_{0}-M_{w}(t)}{\lambda_{1}} .
\end{aligned}
$$

We use the relation between the classical derivative and fractal derivative. Then, we obtain:

$$
\begin{aligned}
& { }_{c}^{C F} D_{t}^{\alpha} M_{u}(t)=\tau t^{\tau-1}\left(\vartheta_{0} M_{v}(t)-\frac{M_{u}(t)}{\lambda_{2}}\right) . \\
& { }_{c}^{C F} D_{t}^{\alpha} M_{v}(t)=\tau t^{\tau-1}\left(-\vartheta_{0} M_{u}(t)-\frac{M_{v}(t)}{\lambda_{2}}\right) . \\
& { }_{c}^{C F} D_{t}^{\alpha} M_{w}(t)=\tau t^{\tau-1}\left(\frac{M_{0}-M_{w}(t)}{\lambda_{1}}\right) .
\end{aligned}
$$

For simplicity, we define:

$$
\begin{aligned}
& A\left(t, M_{u}, M_{v}, M_{w}\right)=\tau t^{\tau-1}\left(\vartheta_{0} M_{v}(t)-\frac{M_{u}(t)}{\lambda_{2}}\right) . \\
& B\left(t, M_{u}, M_{v}, M_{w}\right)=\tau t^{\tau-1}\left(-\vartheta_{0} M_{u}(t)-\frac{M_{v}(t)}{\lambda_{2}}\right) . \\
& C\left(t, M_{u}, M_{v}, M_{w}\right)=\tau t^{\tau-1}\left(\frac{M_{0}-M_{w}(t)}{\lambda_{1}}\right) .
\end{aligned}
$$

Then, we obtain:

$$
\begin{aligned}
& { }_{c}^{C F} D_{t}^{\alpha} M_{u}(t)=A\left(t, M_{u}, M_{v}, M_{w}\right) . \\
& { }_{C}^{C F} D_{t}^{\alpha} M_{v}(t)=B\left(t, M_{u}, M_{v}, M_{w}\right) . \\
& { }_{c}^{C F} D_{t}^{\alpha} M_{w}(t)=C\left(t, M_{u}, M_{v}, M_{w}\right) .
\end{aligned}
$$

Applying the CF integral gives:

$$
\begin{aligned}
M_{u}(t)-M_{u}(0) & =\frac{1-\alpha}{M(\alpha)} A\left(t, M_{u}, M_{v}, M_{w}\right)+\frac{\alpha}{M(\alpha)} \int_{0}^{t} A\left(\tau, M_{u}, M_{v}, M_{w}\right) d \tau . \\
M_{v}(t)-M_{v}(0) & =\frac{1-\alpha}{M(\alpha)} B\left(t, M_{u}, M_{v}, M_{w}\right)+\frac{\alpha}{M(\alpha)} \int_{0}^{t} B\left(\tau, M_{u}, M_{v}, M_{w}\right) d \tau . \\
M_{w}(t)-M_{w}(0) & =\frac{1-\alpha}{M(\alpha)} C\left(t, M_{u}, M_{v}, M_{w}\right)+\frac{\alpha}{M(\alpha)} \int_{0}^{t} C\left(\tau, M_{u}, M_{v}, M_{w}\right) d \tau .
\end{aligned}
$$

We discretize these equations at $t_{n+1}$ and $t_{n}$ as:

$$
\begin{aligned}
M_{u}^{n+1} & =M_{u}^{0}+\frac{1-\alpha}{M(\alpha)} A\left(t_{n}, M_{u}^{n}, M_{v}^{n}, M_{w}^{n}\right)+\frac{\alpha}{M(\alpha)} \int_{0}^{t_{n+1}} A\left(\tau, M_{u}, M_{v}, M_{w}\right) d \tau . \\
M_{v}^{n+1} & =M_{v}^{0}+\frac{1-\alpha}{M(\alpha)} B\left(t_{n}, M_{u}^{n}, M_{v}^{n}, M_{w}^{n}\right)+\frac{\alpha}{M(\alpha)} \int_{0}^{t_{n+1}} B\left(\tau, M_{u}, M_{v}, M_{w}\right) d \tau . \\
M_{w}^{n+1} & =M_{w}^{0}+\frac{1-\alpha}{M(\alpha)} C\left(t_{n}, M_{u}^{n}, M_{v}^{n}, M_{w}^{n}\right)+\frac{\alpha}{M(\alpha)} \int_{0}^{t_{n+1}} C\left(\tau, M_{u}, M_{v}, M_{w}\right) d \tau .
\end{aligned}
$$


and:

$$
\begin{aligned}
M_{u}^{n} & =M_{u}^{0}+\frac{1-\alpha}{M(\alpha)} A\left(t_{n-1}, M_{u}^{n-1}, M_{v}^{n-1}, M_{w}^{n-1}\right)+\frac{\alpha}{M(\alpha)} \int_{0}^{t_{n}} A\left(\tau, M_{u}, M_{v}, M_{w}\right) d \tau . \\
M_{v}^{n} & =M_{v}^{0}+\frac{1-\alpha}{M(\alpha)} B\left(t_{n-1}, M_{u}^{n-1}, M_{v}^{n-1}, M_{w}^{n-1}\right)+\frac{\alpha}{M(\alpha)} \int_{0}^{t_{n}} B\left(\tau, M_{u}, M_{v}, M_{w}\right) d \tau . \\
M_{w}^{n} & =M_{w}^{0}+\frac{1-\alpha}{M(\alpha)} C\left(t_{n-1}, M_{u}^{n-1}, M_{v}^{n-1}, M_{w}^{n-1}\right)+\frac{\alpha}{M(\alpha)} \int_{0}^{t_{n}} C\left(\tau, M_{u}, M_{v}, M_{w}\right) d \tau .
\end{aligned}
$$

Then, we obtain:

$$
\begin{aligned}
M_{u}^{n+1} & =M_{u}^{n}+\frac{1-\alpha}{M(\alpha)}\left(A\left(t_{n}, M_{u}^{n}, M_{v}^{n}, M_{w}\right)-A\left(t_{n-1}, M_{u}^{n-1}, M_{v}^{n-1}, M_{w}^{n-1}\right)\right)+\frac{\alpha}{M(\alpha)} \int_{t_{n}}^{t_{n+1}} A\left(\tau, M_{u}, M_{v}, M_{w}\right) d \tau . \\
M_{v}^{n+1} & =M_{v}^{n}+\frac{1-\alpha}{M(\alpha)}\left(B\left(t_{n}, M_{u}^{n}, M_{v}^{n}, M_{w}\right)-B\left(t_{n-1}, M_{u}^{n-1}, M_{v}^{n-1}, M_{w}^{n-1}\right)\right) . \\
M_{w}^{n+1} & =M_{w}^{n}+\frac{1-\alpha}{M(\alpha)}\left(C\left(t_{n}, M_{u}^{n}, M_{v}^{n}, M_{w}\right)-C\left(t_{n-1}, M_{u}^{n-1}, M_{v}^{n-1}, M_{w}^{n-1}\right)\right)+\frac{\alpha}{M(\alpha)} \int_{t_{n}}^{t_{n+1}} C\left(\tau, M_{u}, M_{v}, M_{w}\right) d \tau .
\end{aligned}
$$

We use the two-step Lagrange polynomial and obtain:

$$
\begin{aligned}
M_{u}^{n+1}= & M_{u}^{n}+\frac{1-\alpha}{M(\alpha)}\left(A\left(t_{n}, M_{u}^{n}, M_{v}^{n}, M_{w}^{n}\right)-A\left(t_{n-1}, M_{u}^{n-1}, M_{v}^{n-1}, M_{w}^{n-1}\right)\right) \\
& +\frac{\alpha}{M(\alpha)}\left(\frac{3 h}{2} A\left(t_{n}, M_{u}^{n}, M_{v}^{n}, M_{w}^{n}\right)-\frac{h}{2} A\left(t_{n-1}, M_{u}^{n-1}, M_{v}^{n-1}, M_{w}^{n-1}\right)\right) . \\
M_{v}^{n+1}= & M_{v}^{n}+\frac{1-\alpha}{M(\alpha)}\left(B\left(t_{n}, M_{u}^{n}, M_{v}^{n}, M_{w}^{n}\right)-B\left(t_{n-1}, M_{u}^{n-1}, M_{v}^{n-1}, M_{w}^{n-1}\right)\right) \\
& +\frac{\alpha}{M(\alpha)}\left(\frac{3 h}{2} B\left(t_{n}, M_{u}^{n}, M_{v}^{n}, M_{w}^{n}\right)-\frac{h}{2} B\left(t_{n-1}, M_{u}^{n-1}, M_{v}^{n-1}, M_{w}^{n-1}\right)\right) . \\
M_{w}^{n+1}= & M_{w}^{n}+\frac{1-\alpha}{M(\alpha)}\left(C\left(t_{n}, M_{u}^{n}, M_{v}^{n}, M_{w}^{n}\right)-A\left(t_{n-1}, M_{u}^{n-1}, M_{v}^{n-1}, M_{w}^{n-1}\right)\right) \\
& +\frac{\alpha}{M(\alpha)}\left(\frac{3 h}{2} C\left(t_{n}, M_{u}^{n}, M_{v}^{n}, M_{w}^{n}\right)-\frac{h}{2} C\left(t_{n-1}, M_{u}^{n-1}, M_{v}^{n-1}, M_{w}^{n-1}\right)\right) .
\end{aligned}
$$

\section{Numerical simulations}

We establish the numerical results for magnetization components $\left(M_{u}, M_{v}, M_{w}\right)$ at distinct values of $\alpha$ and $\tau$ by taking $\alpha$ as fractional order and $\tau$ as fractal dimension. We obtain the numerical simulations of the proposed model for different values of $\alpha$ and $\tau$. We analyze the model with three different kernels- power law, exponential decay law and MittagLeffler law. In the Figures 1-9, we use the Mittag-Leffler type kernel. In Figures 1-3, we choose the fractal dimension $\tau=1$ and check the effect of the fractional order in these figures. Similarly, in Figures 4-6 and Figures 7-9, we set the fractal dimension $\tau=0.98$ and $\tau=0.9$ respectively. Also, we use the power law, exponential decay type kernels in the Figures 10-18 and Figures 19-27 respectively to illustrate the significant features of magnitization components of fractal-fractional Bloch equations with respect to time $t$.

\section{Conclusions}

In this manuscript, we studied new aspects of Bloch equations by considering the fractal-fractional derivatives. The solutions corresponding to magnetization components $\left(M_{u}, M_{v}, M_{w}\right)$ of fractal-fractional Bloch equations were evalu- 


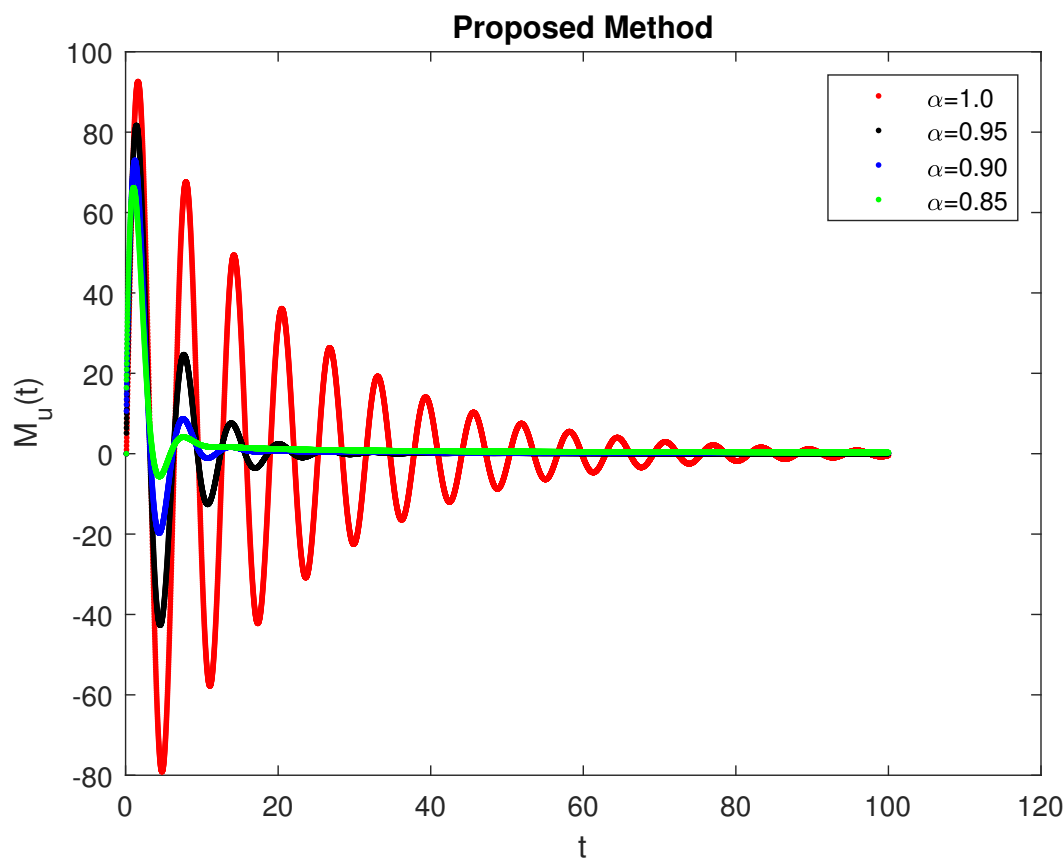

Figure 1: Numerical simulations for $\tau=1$ for $A B C$ derivative.

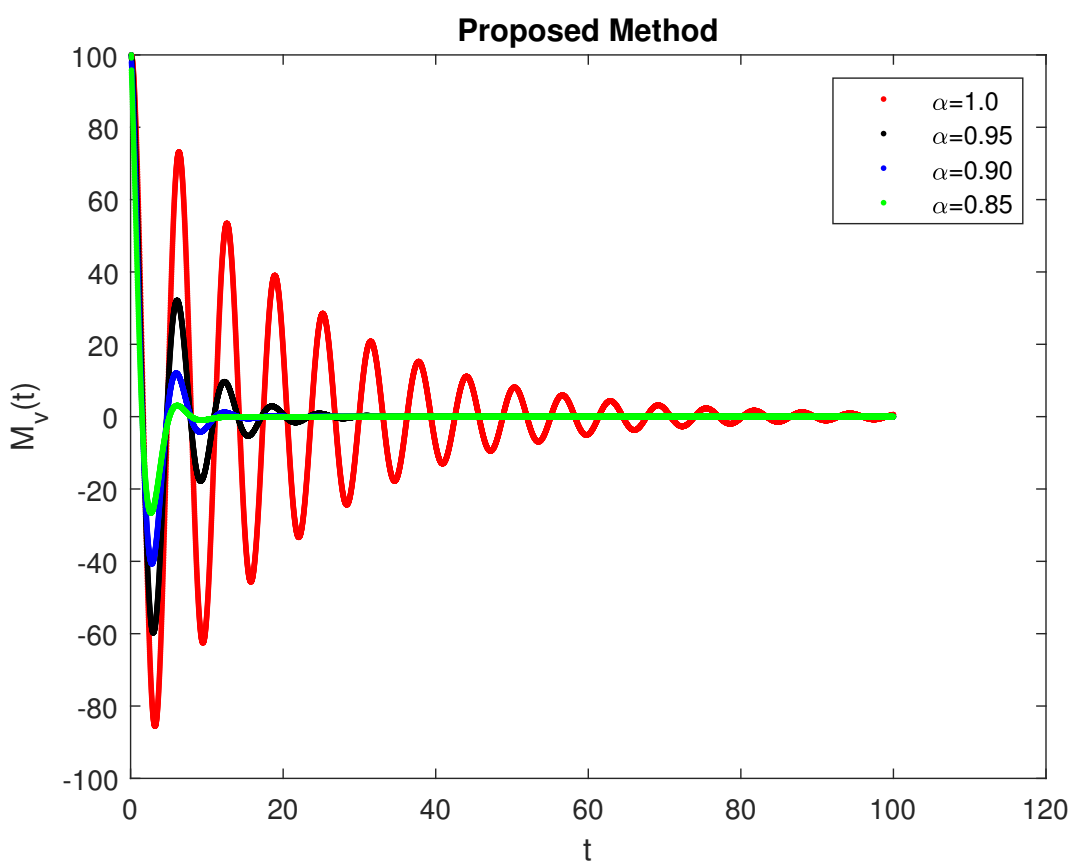

Figure 2: Numerical simulations for $\tau=1$ for $A B C$ derivative. 


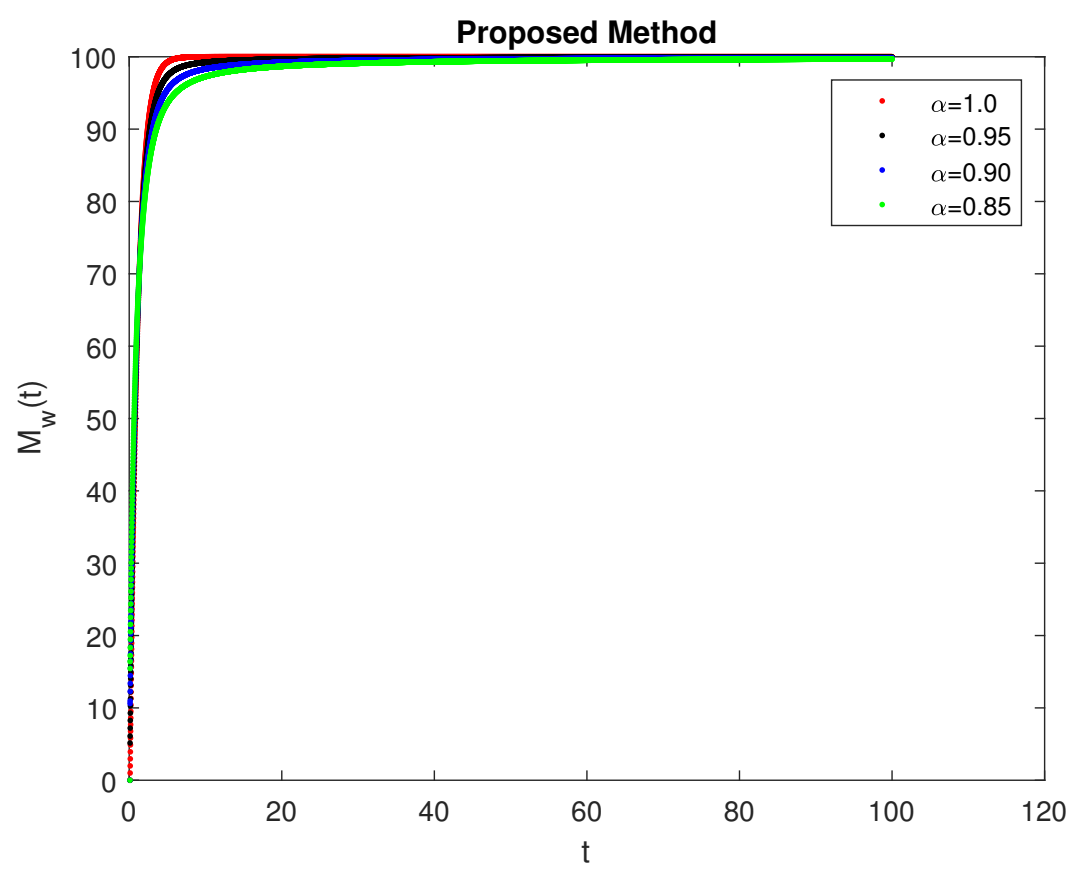

Figure 3: Numerical simulations for $\tau=1$ for $A B C$ derivative.

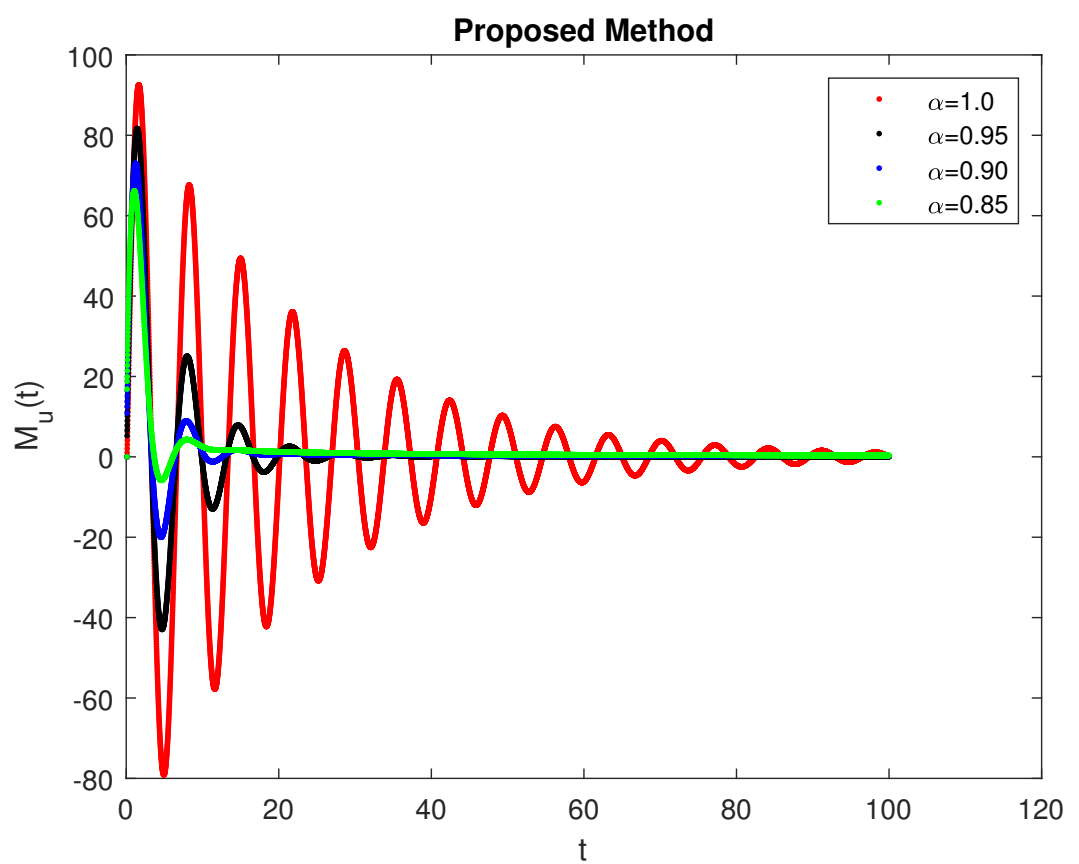

Figure 4: Numerical simulations for $\tau=0.98$ for $A B C$ derivative. 


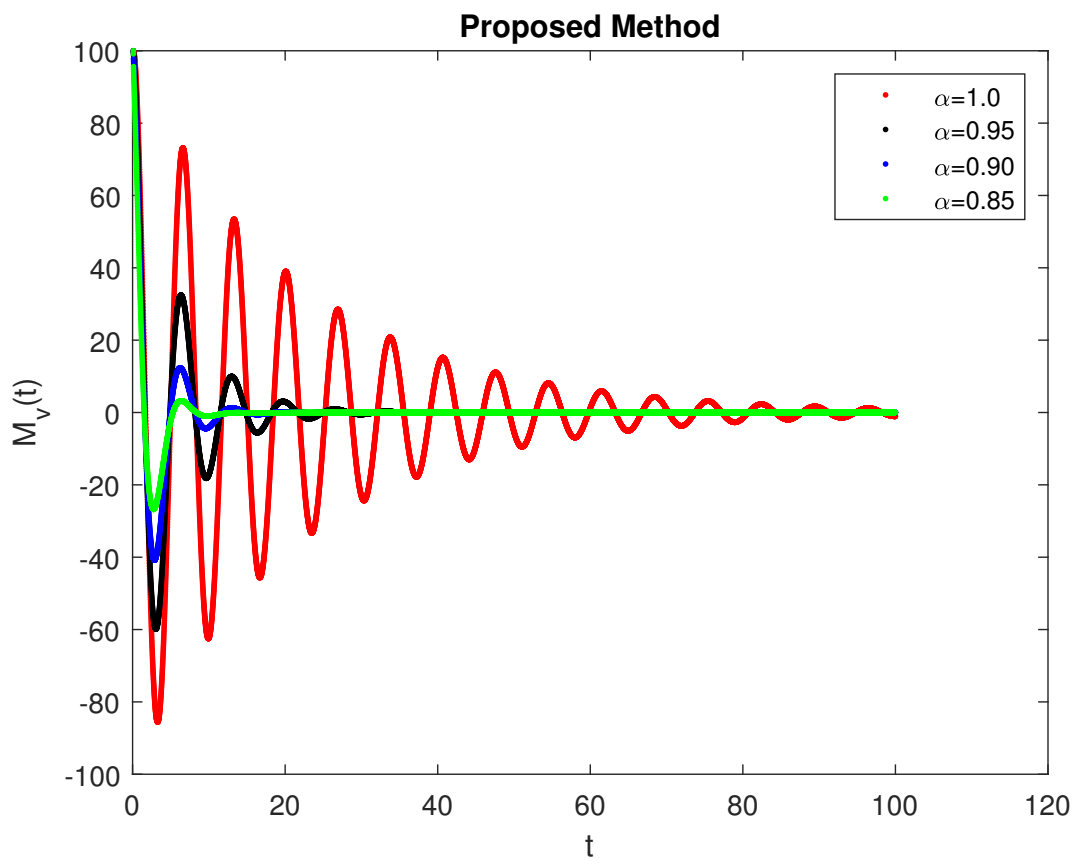

Figure 5: Numerical simulations for $\tau=0.98$ for $A B C$ derivative.

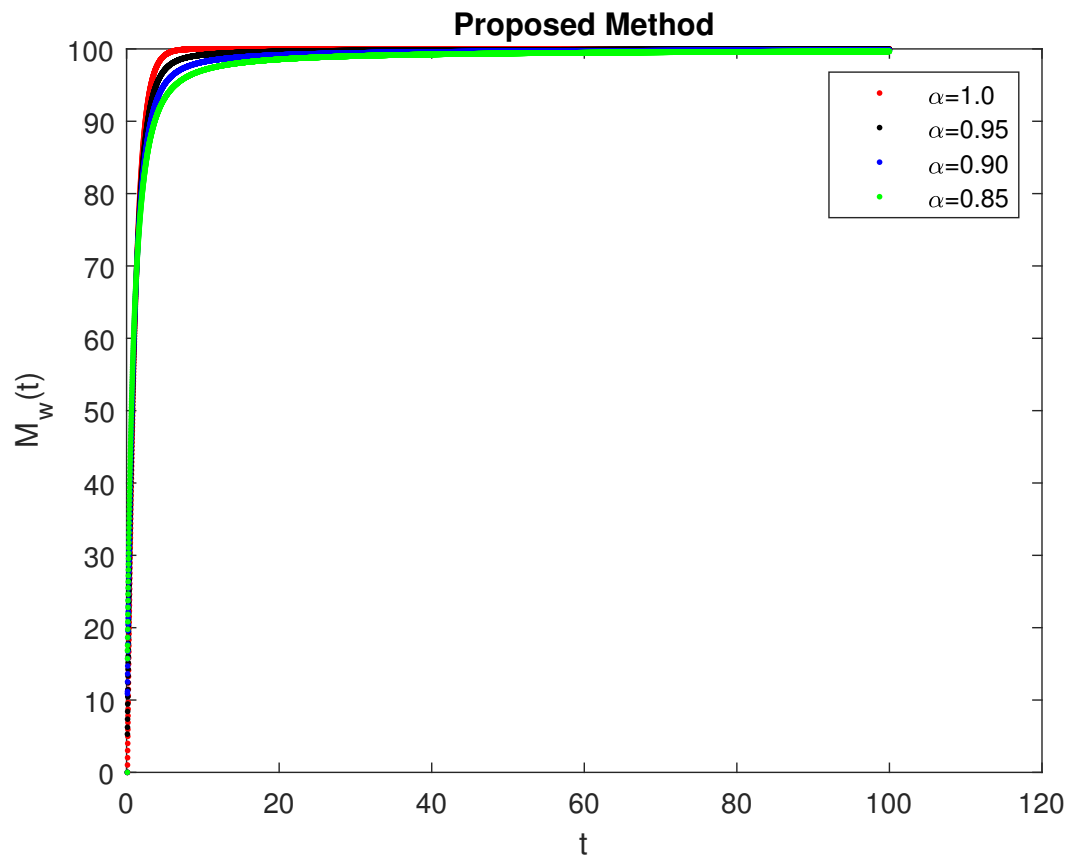

Figure 6: Numerical simulations for $\tau=0.98$ for $A B C$ derivative. 


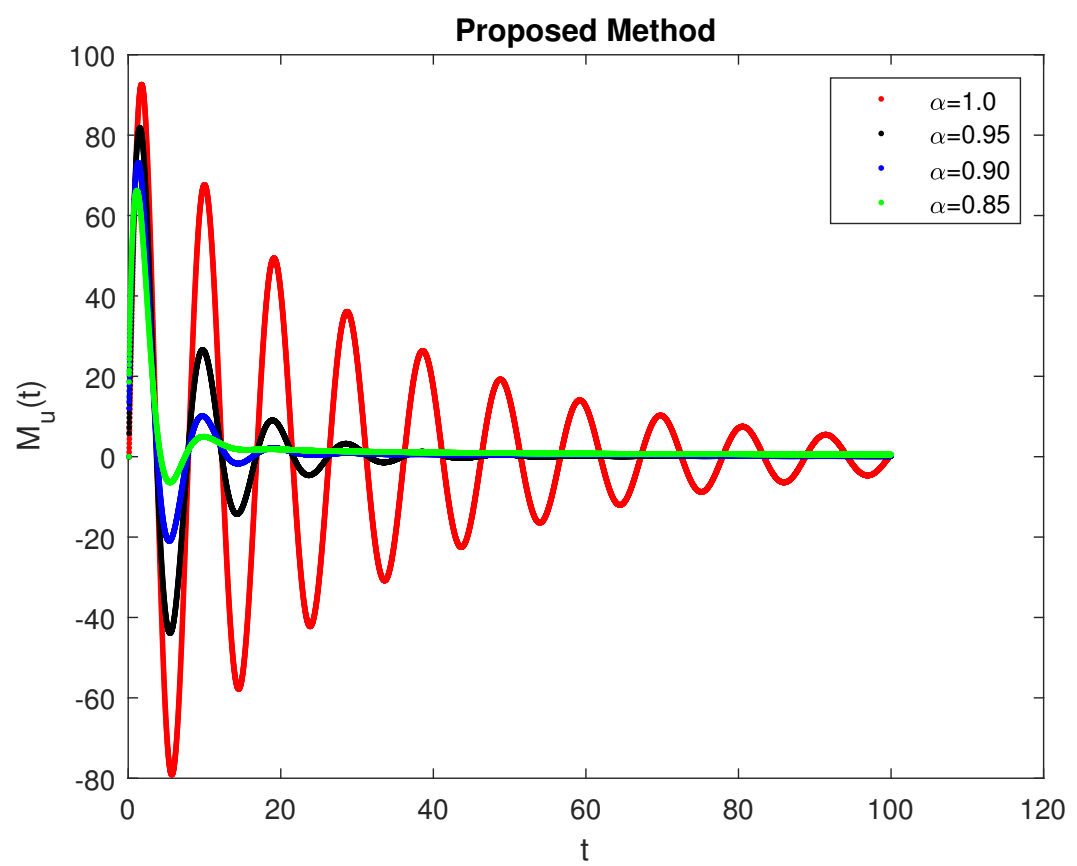

Figure 7: Numerical simulations for $\tau=0.98$ for $\mathrm{ABC}$ derivative.

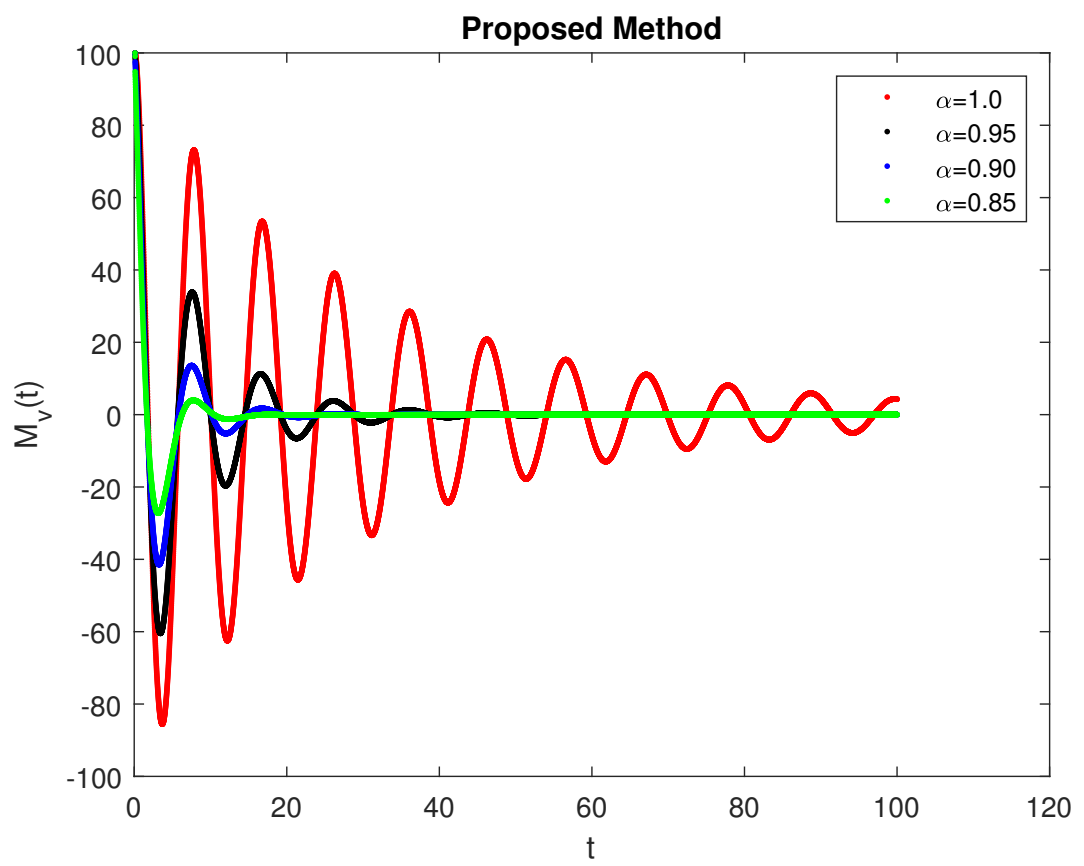

Figure 8: Numerical simulations for $\tau=0.9$ for $A B C$ derivative. 


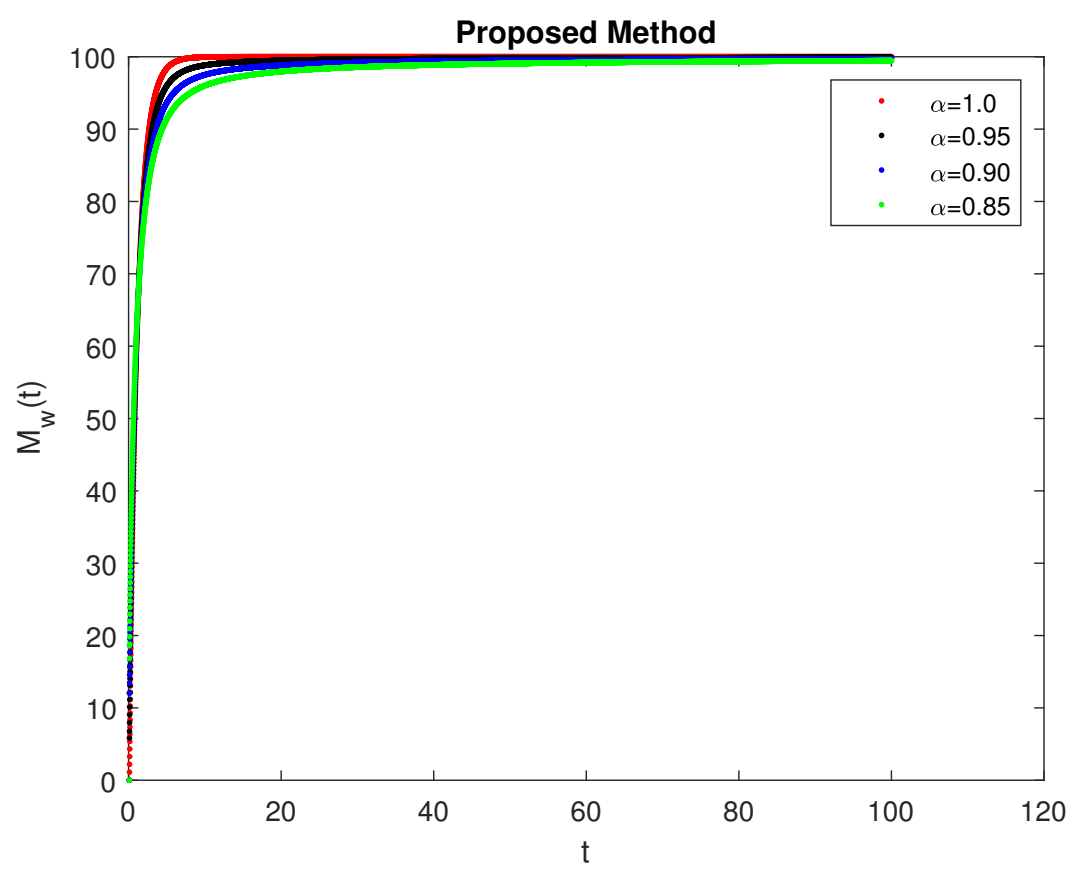

Figure 9: Numerical simulations for $\tau=0.9$ for $A B C$ derivative.

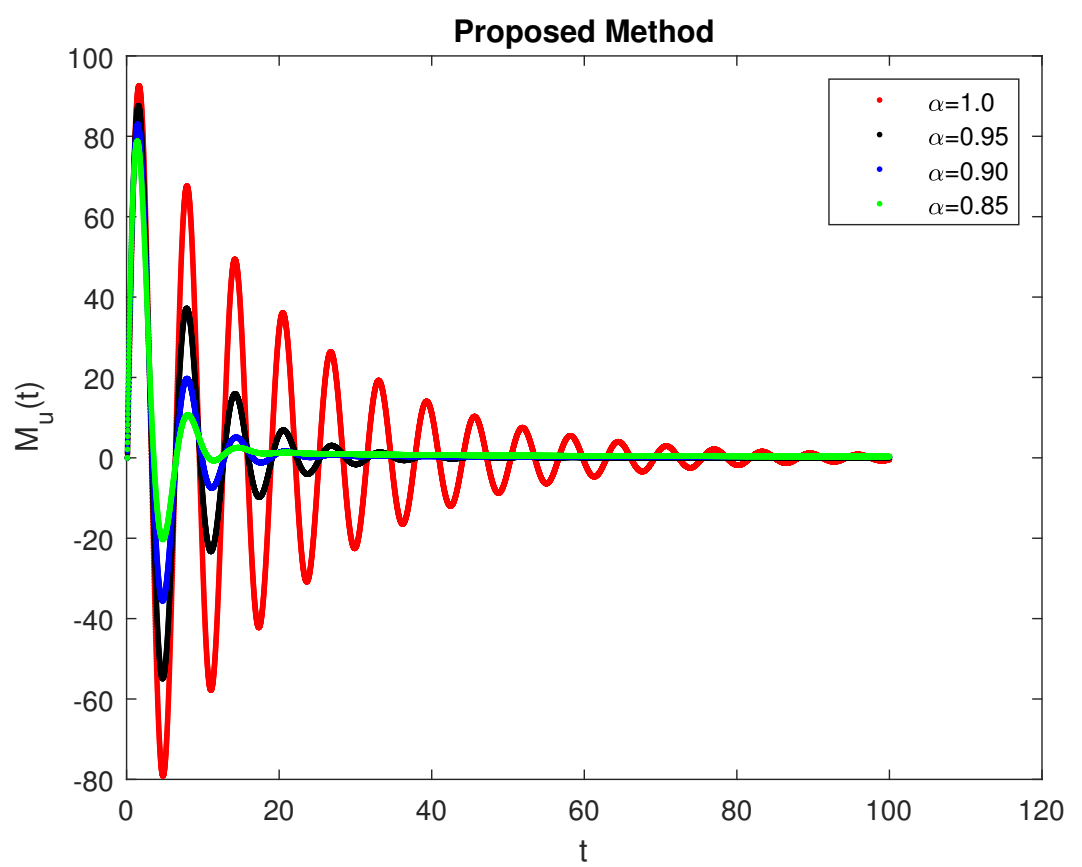

Figure 10: Numerical simulations for $\tau=1$ for Caputo derivative. 


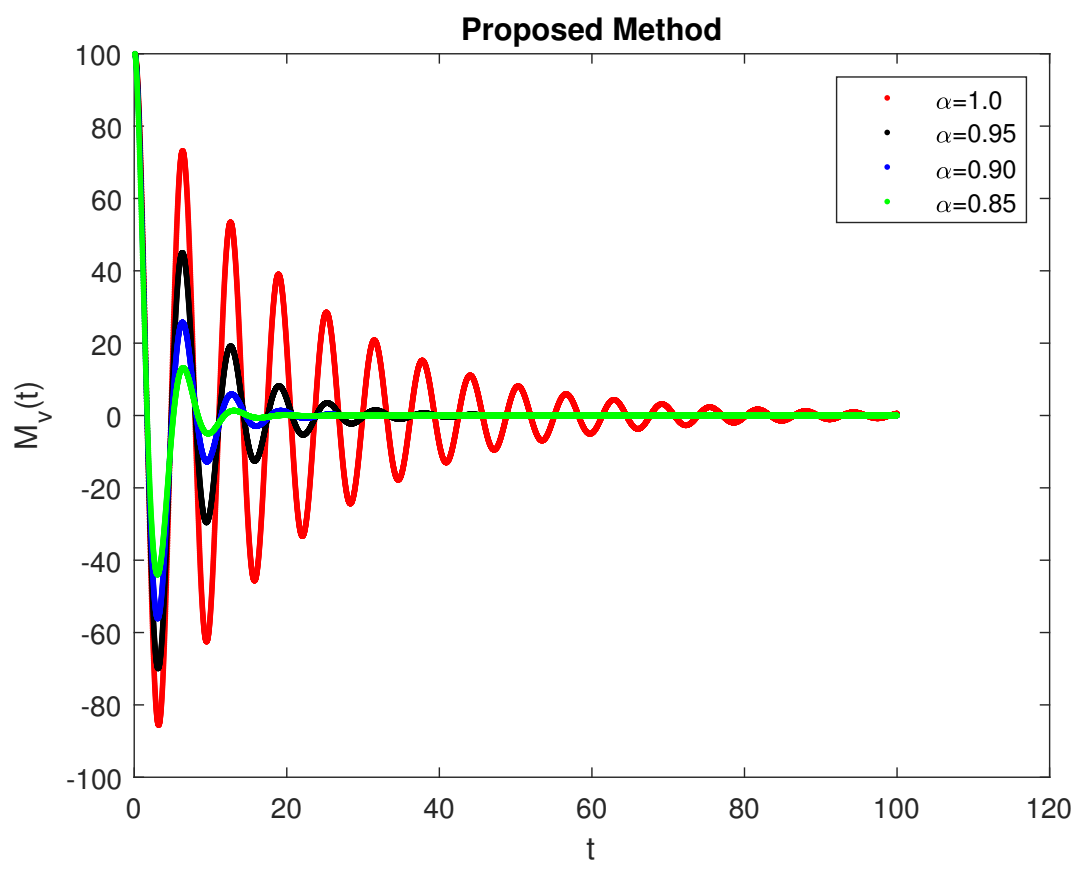

Figure 11: Numerical simulations for $\tau=1$ for Caputo derivative.

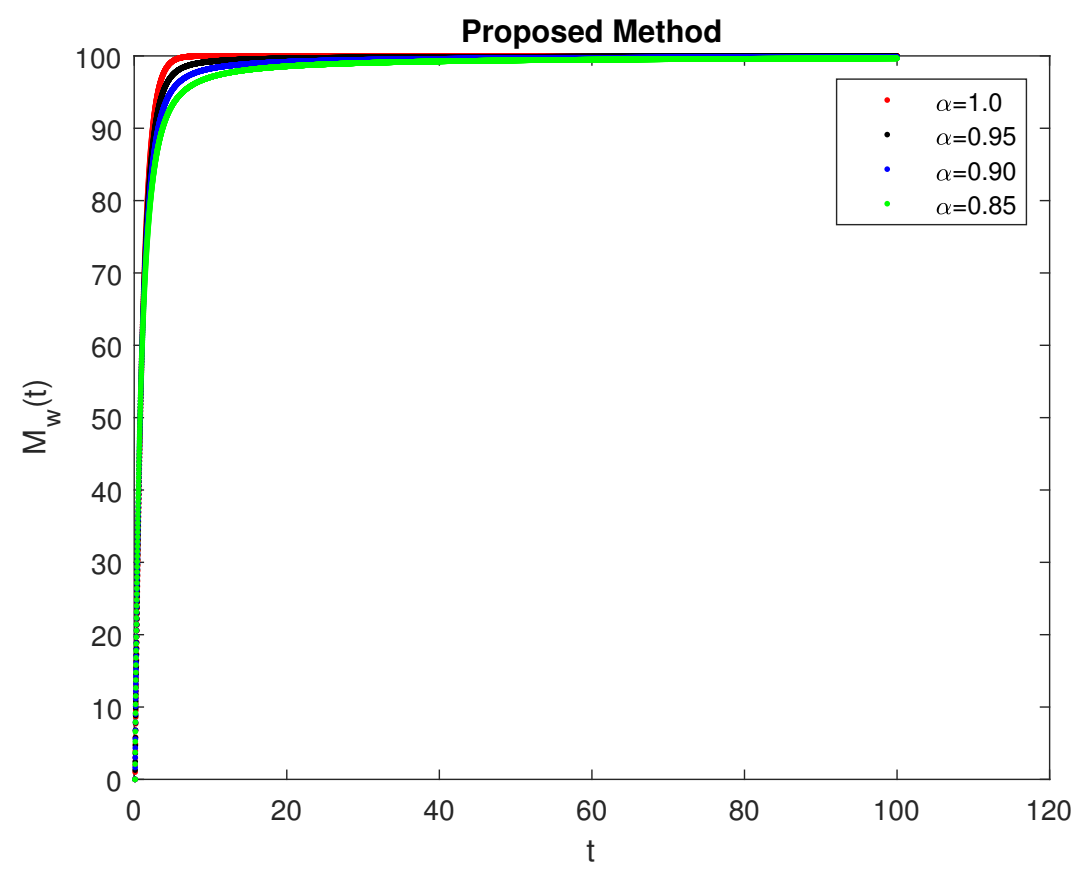

Figure 12: Numerical simulations for $\tau=1$ for Caputo derivative. 


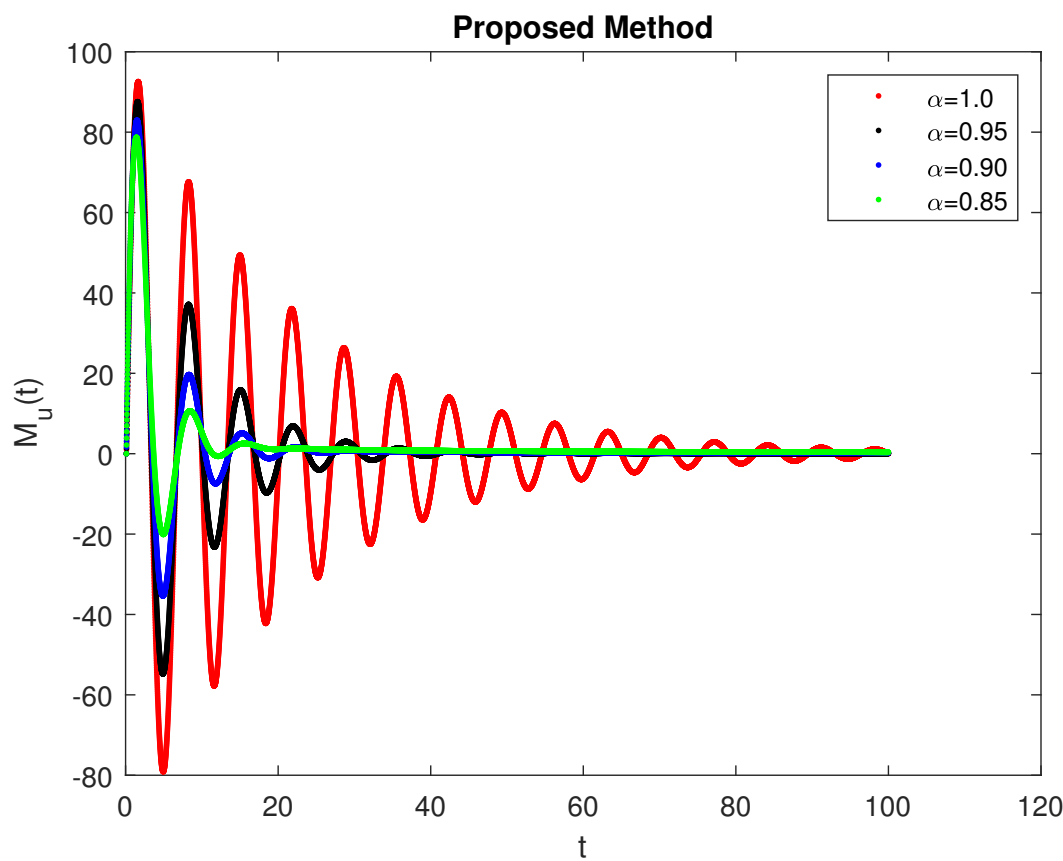

Figure 13: Numerical simulations for $\tau=0.98$ for Caputo derivative.

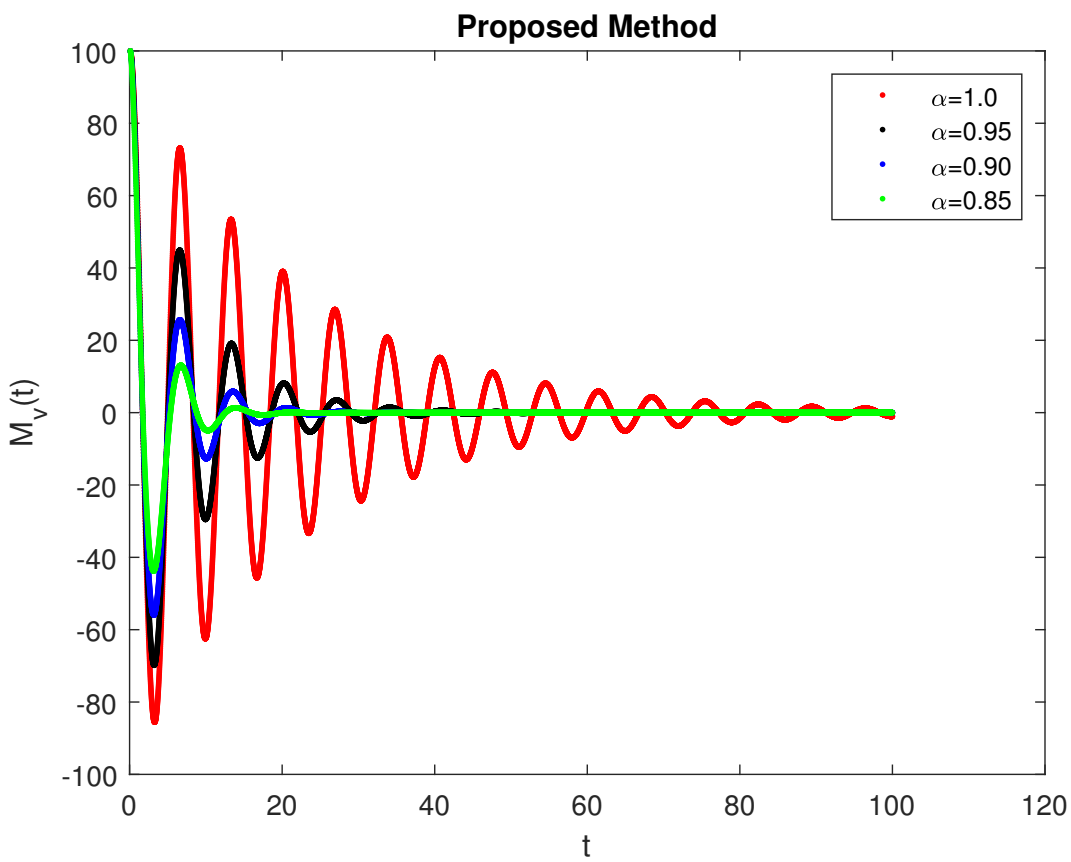

Figure 14: Numerical simulations for $\tau=0.98$ for Caputo derivative. 


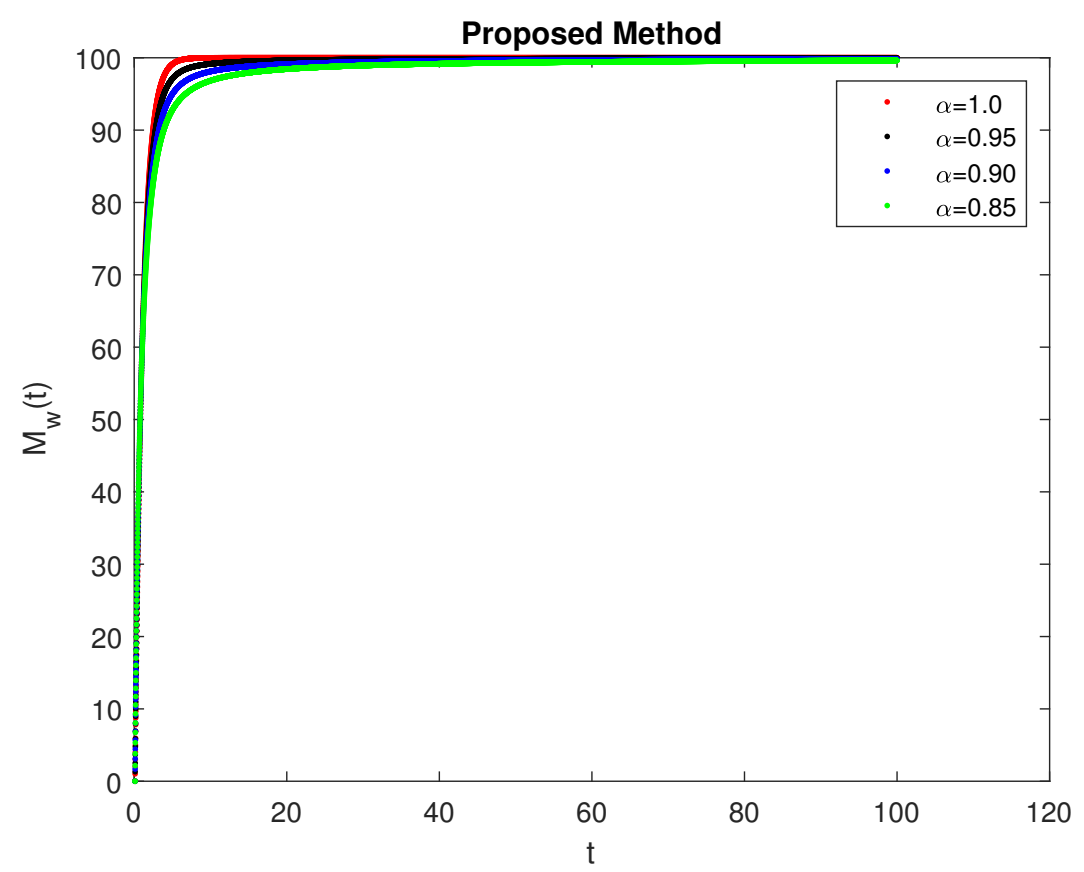

Figure 15: Numerical simulations for $\tau=0.98$ for Caputo derivative.

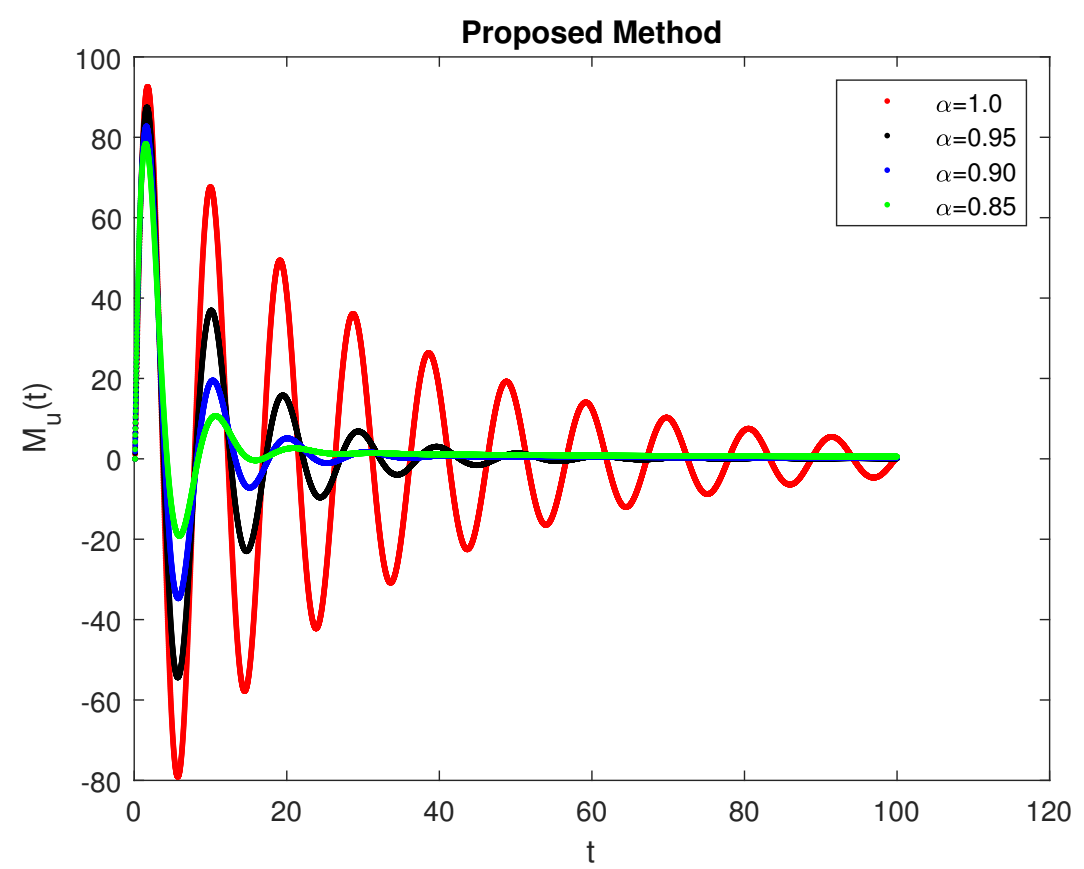

Figure 16: Numerical simulations for $\tau=0.98$ for Caputo derivative. 


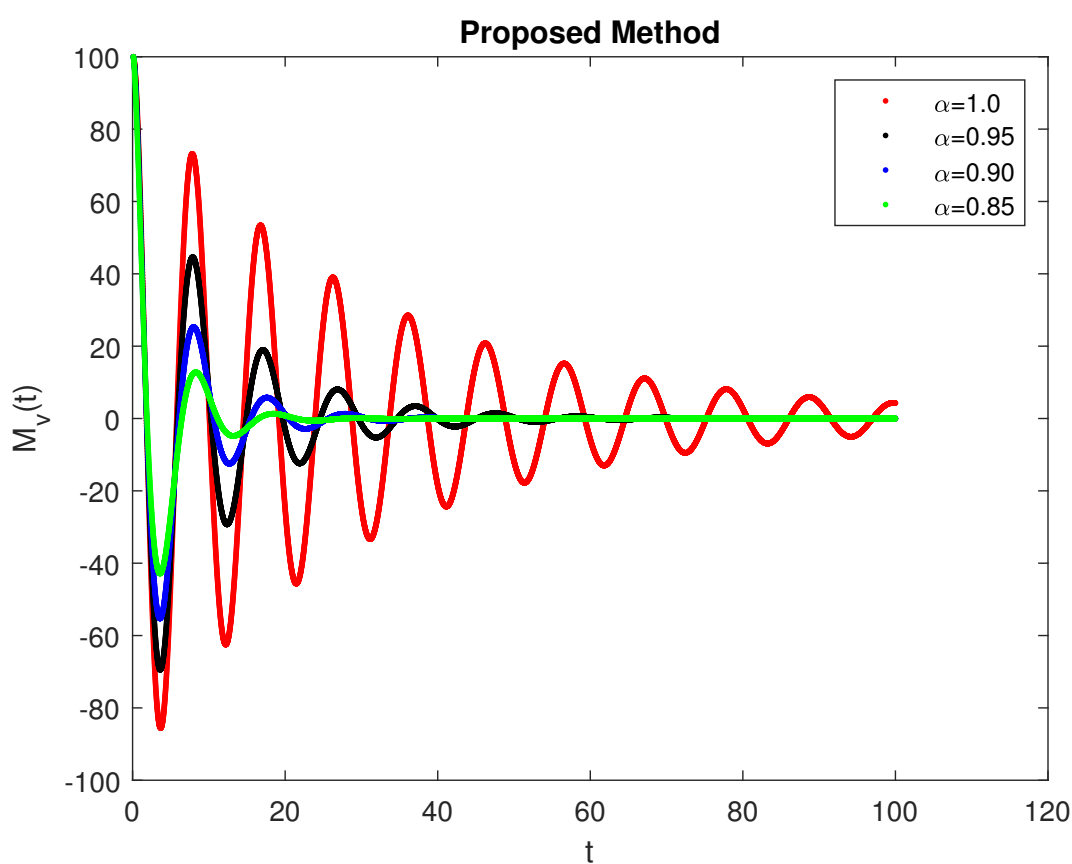

Figure 17: Numerical simulations for $\tau=0.9$ for Caputo derivative.

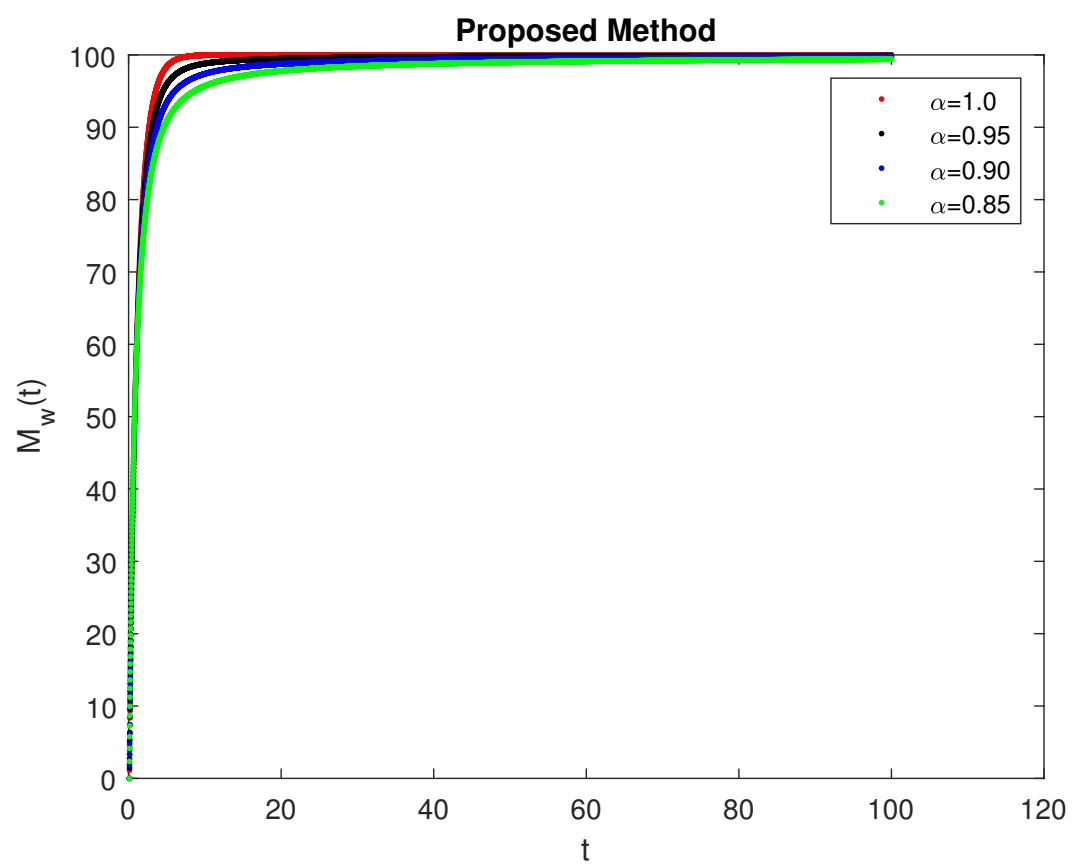

Figure 18: Numerical simulations for $\tau=0.9$ for Caputo derivative. 


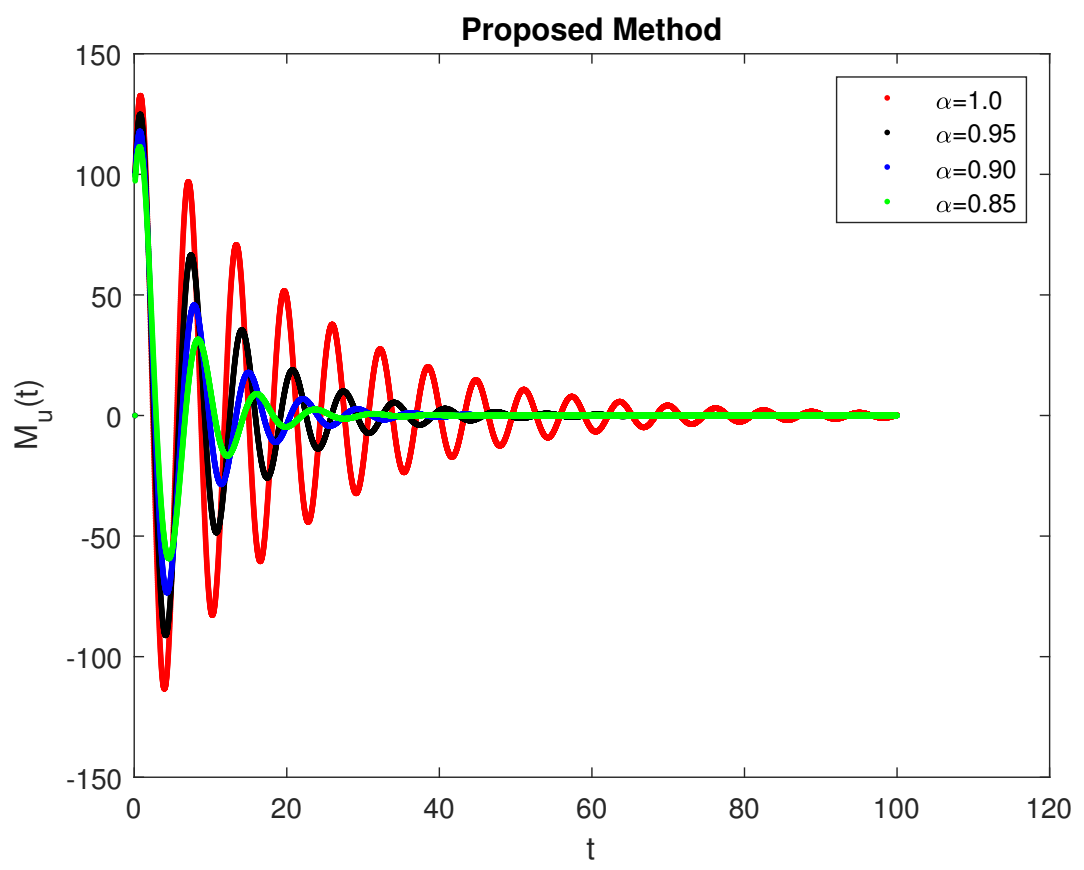

Figure 19: Numerical simulations for $\tau=1$ for Caputo-Fabrizio derivative.

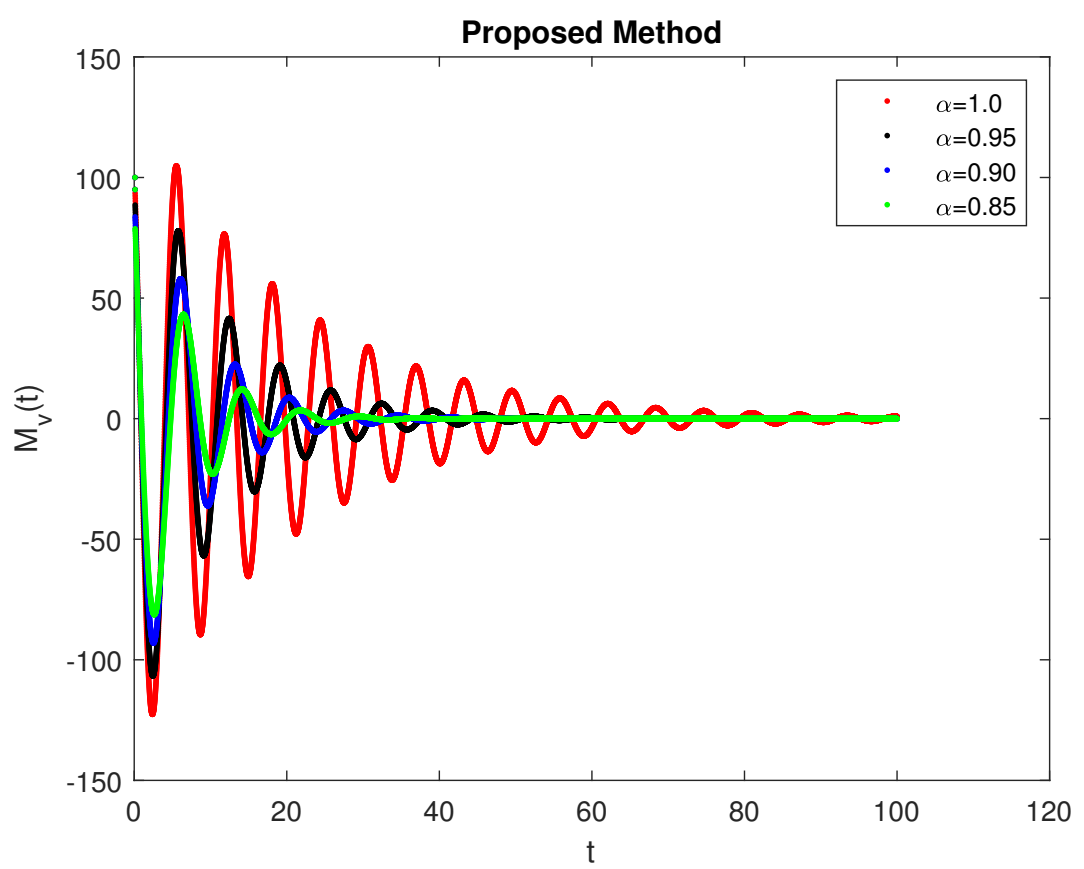

Figure 20: Numerical simulations for $\tau=1$ for Caputo-Fabrizio derivative. 


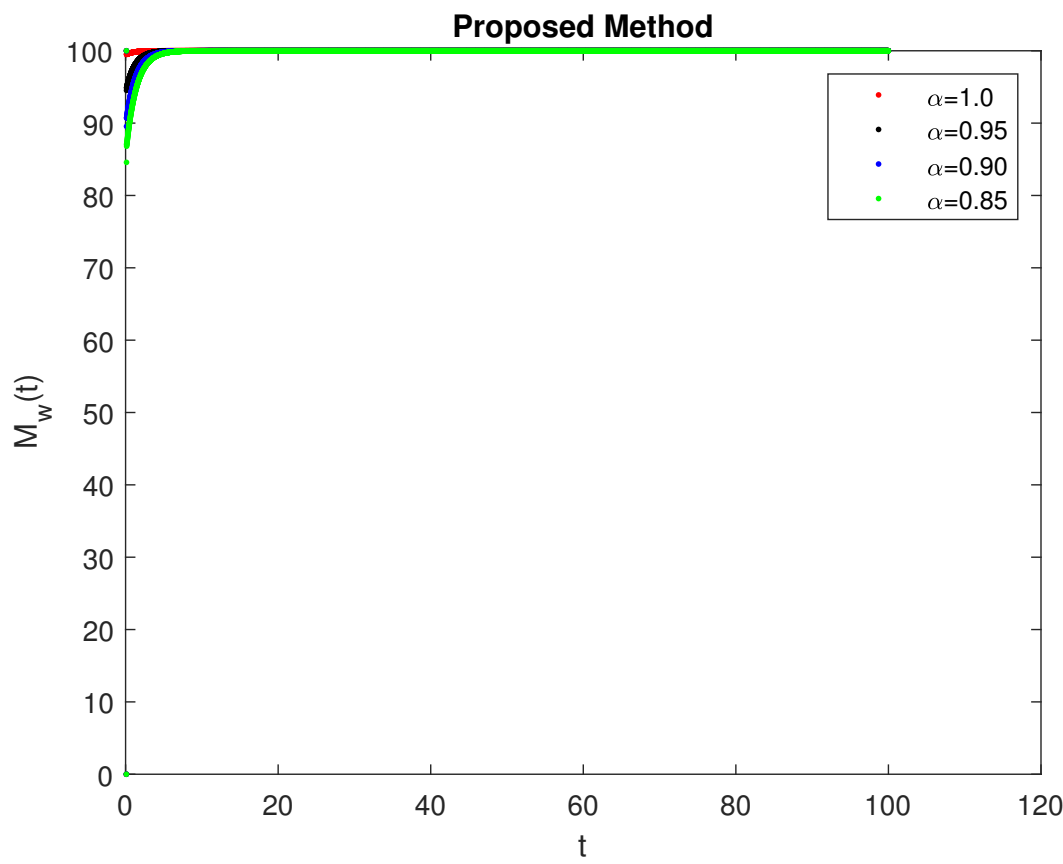

Figure 21: Numerical simulations for $\tau=1$ for Caputo-Fabrizio derivative.

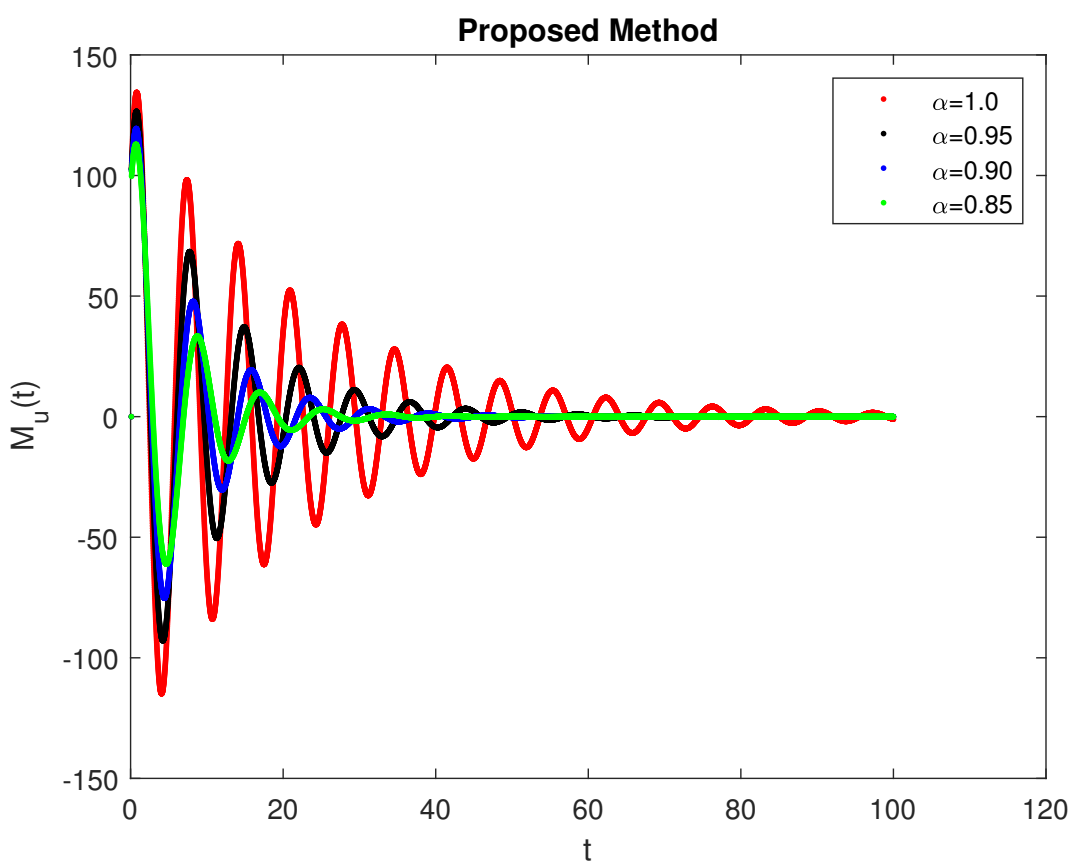

Figure 22: Numerical simulations for $\tau=0.98$ for Caputo-Fabrizio derivative. 


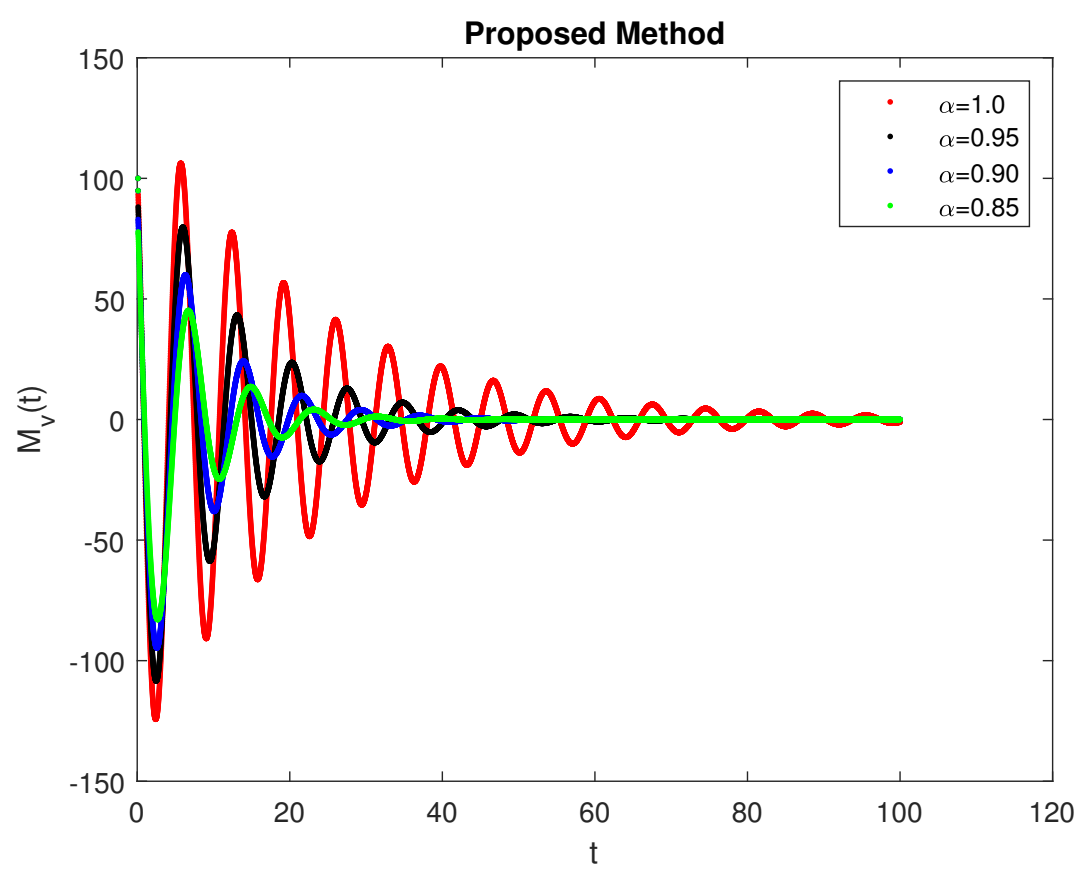

Figure 23: Numerical simulations for $\tau=0.98$ for Caputo-Fabrizio derivative.

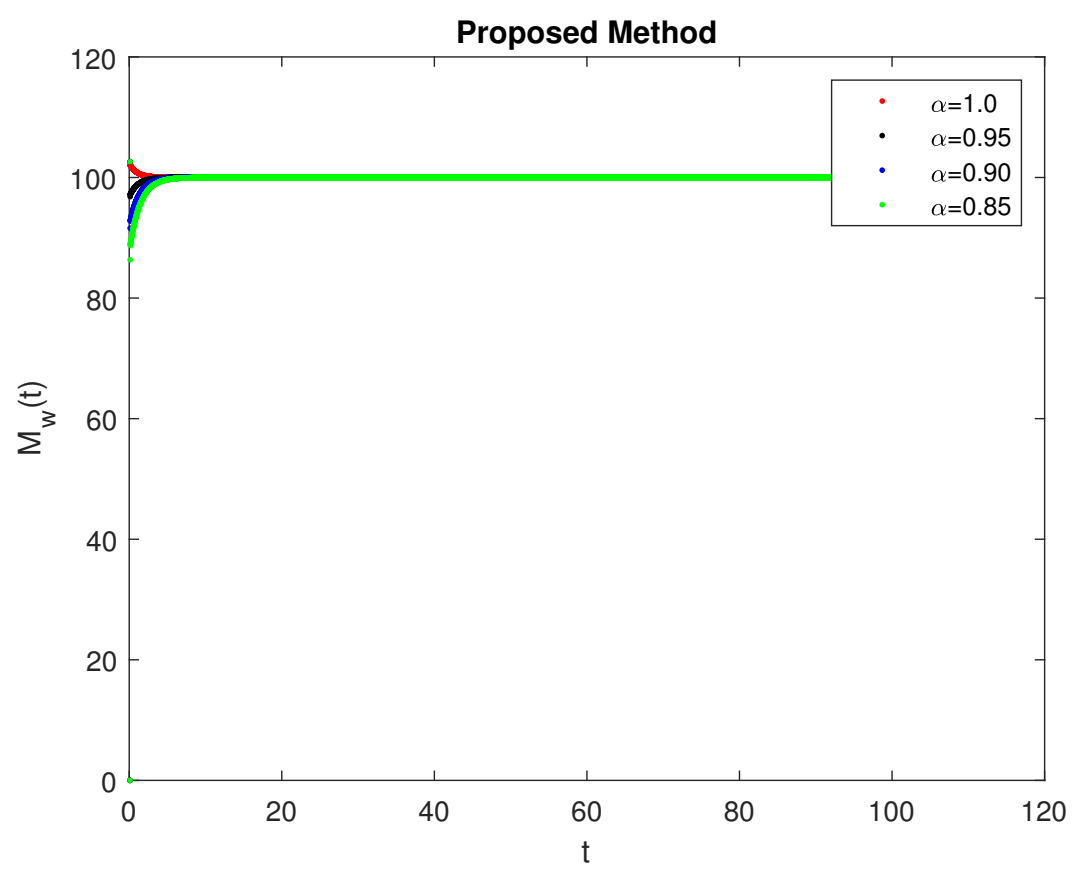

Figure 24: Numerical simulations for $\tau=0.98$ for Caputo-Fabrizio derivative. 


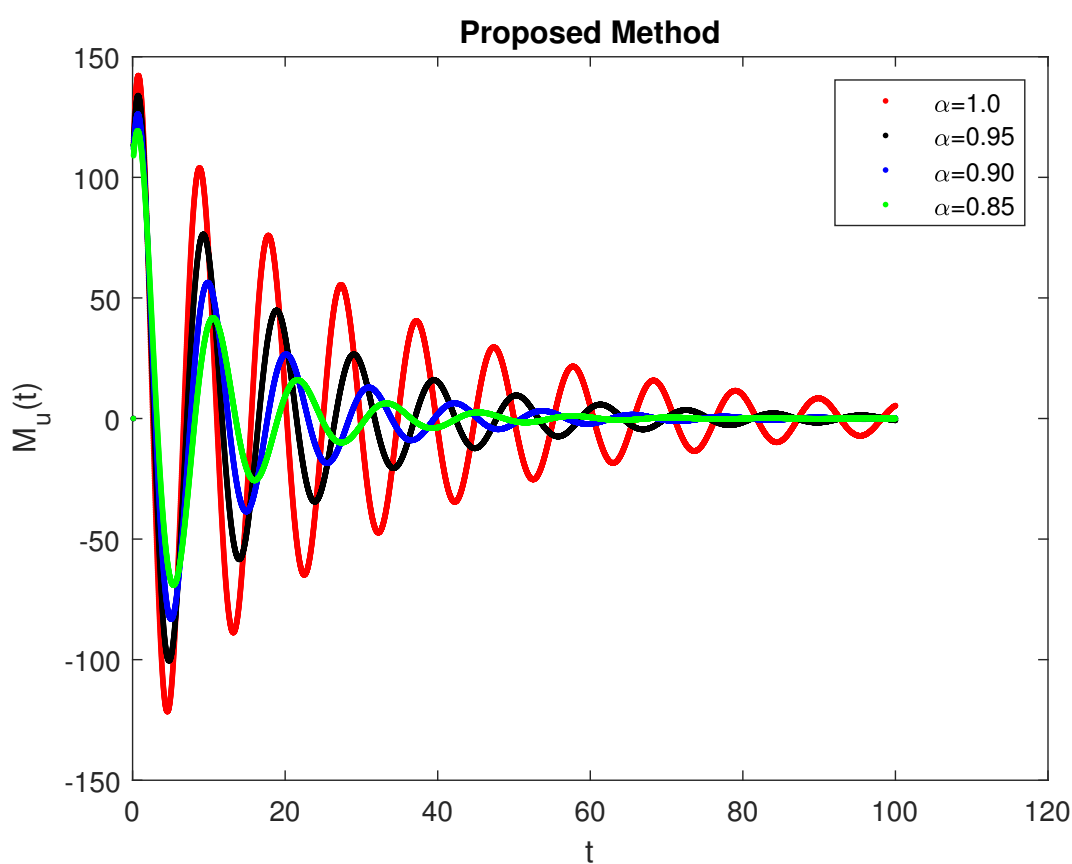

Figure 25: Numerical simulations for $\tau=0.98$ for Caputo-Fabrizio derivative.

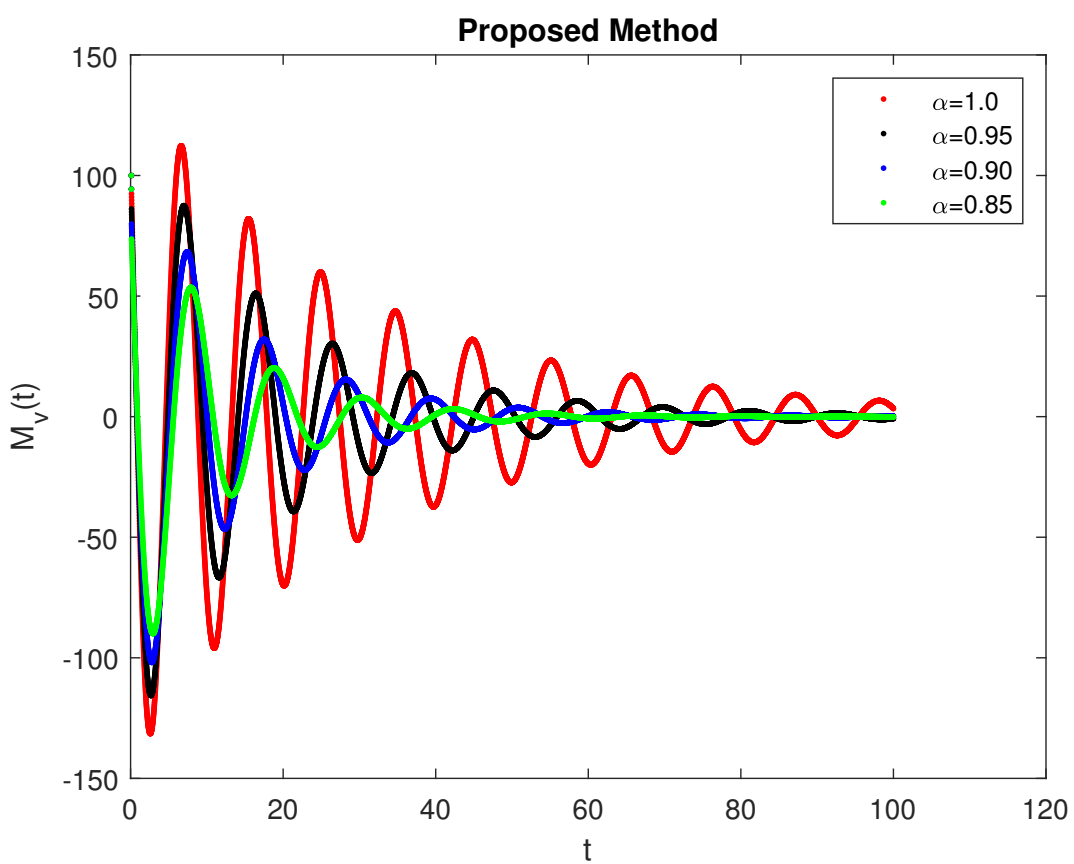

Figure 26: Numerical simulations for $\tau=0.9$ for Caputo-Fabrizio derivative. 


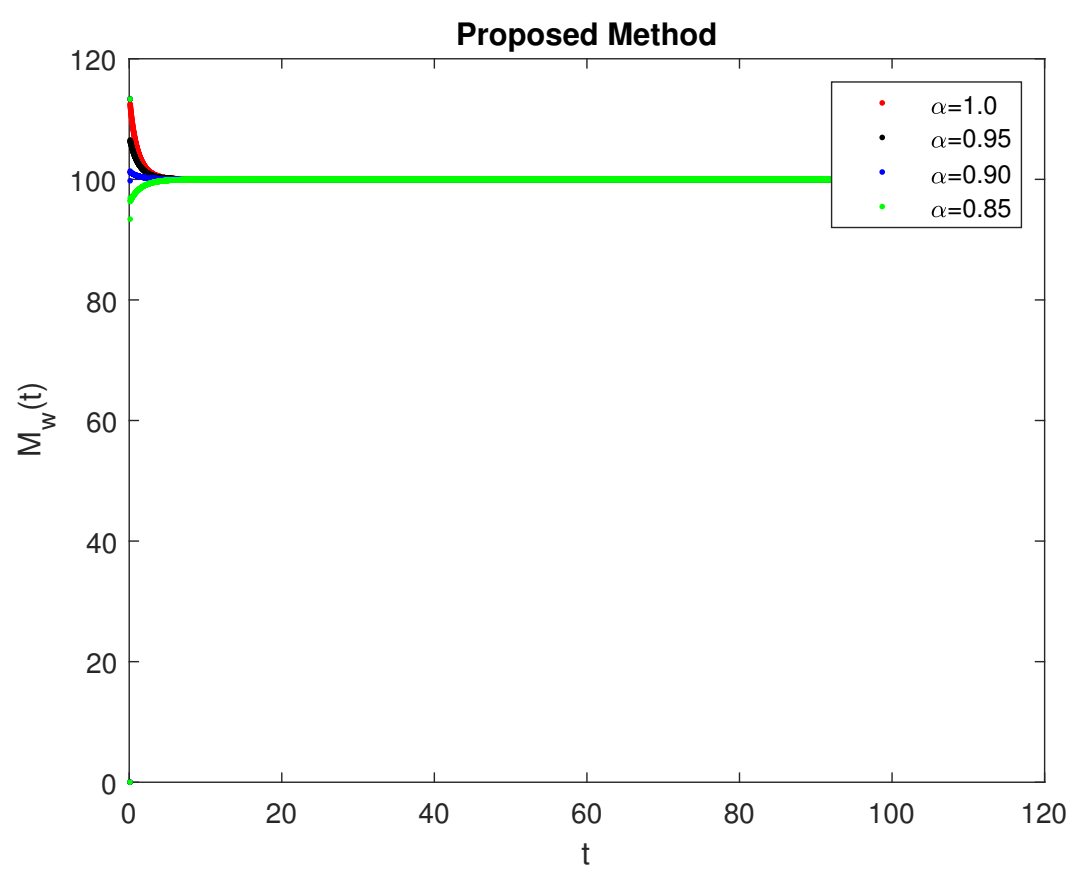

Figure 27: Numerical simulations for $\tau=0.9$ for Caputo-Fabrizio derivative.

ated at distinct values of parameters $\alpha$ and $\tau$ by using descritization and Lagrange's two step numerical scheme. We analyzed the model with three different kernels namely power law, exponential decay and Mittag-Leffler. Additionally, the corresponding graphical representation of the magnetization components $\left(M_{u}, M_{v}, M_{w}\right)$ on several distinct orders were obtained with respect to time $t$.

Funding information: The authors state no funding involved.

Author contributions: All authors have accepted responsibility for the entire content of this manuscript and approved its submission.

Conflict of interest: The authors state no conflict of interest.

\section{References}

[1] Singh J, Kumar D, Baleanu D. New Aspects of Fractional Bloch Model Associated with Composite Fractional Derivative. Math Model Nat Phenom. 2021;16:1-10.

[2] Chen W. Time-space fabric underlying anomalous diffusion. Chaos Solitons Fract. 2006;28(4):923-29.

[3] Fan J, He JH. Fractal derivative model for air permeability in hierarchic porous media. Abstr Appl Anal. 2012;2012:1-7.

[4] Fan J, Shang X. Fractal heat transfer in wool fiber hierarchy. Heat Transf Res. 2013;44(5):399-407.

[5] Fei DD, Liu FJ, Cui QN, He JH. Fractal approach to heat transfer in silkworm cocoon hierarchy. Therm Sci. 2013;17(5):1546-48.

[6] Hu Z, Tu X. A new discrete economic model involving generalized fractal derivative. Adv Differ Equ. 2015;2015:1-11.

[7] Shang X, Wang J, Yang X. Fractal analysis for heat extraction in geothermal system. Therm Sci. 2017;21(1):25-31.

[8] Chen W, Liang Y. New methodologies in fractional and fractal derivatives modeling. Chaos, Solitons Fract. 2017;102:72-77.

[9] Allwright A, Atangana A. Fractal advection-dispersion equation for groundwater transport in fractured aquifers with self-similarities. Eur Phys J Plus. 2018;133(2):1-20.

[10] Prakash A, Kaur H. An efficient hybrid computational technique for solving nonlinear local fractional partial differential equations arising in fractal media. Nonlinear Eng. 2018;7(3):229-35.

[11] Caputo M, Fabrizio M. A new definition of fractional derivative without singular kernel. Prog Fract Differ Appl. 2015;1(2):1-13. 
[12] Atangana A . Fractal-fractional differentiation and integration: connecting fractal calculus and fractional calculus to predict complex system. Chaos, solitons fract. 2017;102:396-406.

[13] Caputo M. Linear models of dissipation whose Q is almost frequency independent-II. Geophys J Int. 1967;13(5):529-39.

[14] Atangana A, Baleanu D. New fractional derivatives with nonlocal and non-singular kernel: theory and application to heat transfer model. Therm Sci. 2016;20:763-69.

[15] Atangana A. Derivative with two fractional orders: A new avenue of investigation toward revolution in fractional calculus. Eur Phys J Plus. 2016;131(10):1-13.

[16] Atangana A. Blind in a commutative world: simple illustrations with functions and chaotic attractors. Chaos, Solitons Fract. 2018;114:347-363.

[17] Singh H, Srivastava HM. Numerical simulation for fractional-order Bloch equation arising in nuclear magnetic resonance by using the Jacobi polynomials. Appl Sci. 2020;10(8):1-18.

[18] Yan H, Chen B, Gore J. Approximate solutions of the Bloch equations for selective excitation. J Magn Reson. 1987;75(1):83-95.

[19] Awojoyogbe B. Analytical solution of the time-dependent Bloch NMR flow equations: a translational mechanical analysis.Phys A: Stat Mech Appl. 2004;339(3-4)437-460.

[20] Atangana A, Akgül A, Owolabi K. Analysis of fractal fractional differential equations. Alex Eng J. 2020;59(3):1117-34.

[21] Toufik M, Atangana A. New numerical approximation of fractional derivative with non-local and non-singular kernel: application to chaotic models. Eur Phys J Plus. 2017;132(10):1-16. 